\title{
Two methods of generalized Ulam-Hyers stability of a quattuorvigintic functional equation in various Banach spaces
}

\author{
John Micheal Rassias ${ }^{1}$, Elumalai Sathya ${ }^{2 *}$ and Mohan Arunkumar ${ }^{3}$
}

\begin{abstract}
In this paper, we introduce and investigate the generalized Ulam - Hyers stability of a quattuorvigintic functional equation in various Banach spaces using two methods.
\end{abstract}

\section{Keywords}

Quattuorvigintic functional equation, generalized Ulam - Hyers stability, Banach space, quasi beta Banach space, fixed point.

\section{AMS Subject Classification}

39B52, 39B72, 39B82.

${ }^{1}$ Pedagogical Department E.E., Section of Mathematics and Informatics,

National and Kapodistrian University of Athens, Athens 15342, Greece.

${ }^{2}$ Department of Mathematics, Shanmuga Industries Arts and Science College, Tiruvannamalai - 606 603, TamilNadu, India.

${ }^{3}$ Department of Mathematics, Department of Mathematics, Government Arts College, Tiruvannamalai - 606 603, TamilNadu, India.

*Corresponding author: ${ }^{1}$ jrassias@primedu.uoa.gr; ${ }^{2}$ sathya24mathematics@gmail.com; ${ }^{3}$ drarun4maths@gmail.com

Article History: Received 22 November 2018; Accepted 09 May 2019

(C)2019 MJM

\section{Contents}



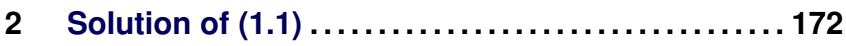

3 Stability Analysis: Banach Space.............175

3.1 Ulam - Hyers Method . . . . . . . . . . . . 175

3.2 Ulam - Radus Method . . . . . . . . . . . . . 177

4 Stability Results : Quasi Beta Banach Spaces ... 179

4.1 Basic Definitions and Notations . . . . . . . . 179

4.2 Ulam - Hyers Method . . . . . . . . . . . . . 179

4.3 Ulam - Radus Method . . . . . . . . . . . . . 185

References 186

\section{Introduction}

In [41], Ulam proposed the universal Ulam stability problem: When is it true that by slightly changing the hypotheses of a theorem one can still assert that the thesis of the theorem remains true or approximately true?. In [18], Hyers gave the first assenting answer to the question of Ulam for additive functional equations on Banach spaces. Hyers result has since then seen many important generalizations, both in terms of the control condition used to define the concept of approximate solution $[2,16,28,35]$. On the other hand, a fixed point alternative method is very important for the solution of the Ulam problem see [10] and [9].

During the last seven decades, the stability problems of a variety of functional equations in quite a lot of spaces have been broadly investigated by number of mathematicians $[3,5$, $8,12,15,17,22,27,32,34,36,39,42]$.

Let us introduce the following quattuorvigintic functional equation

$$
\begin{aligned}
& f(x+12 y)-24 f(x+11 y)+276 f(x+10 y)-2024 f(x+9 y) \\
& +10626 f(x+8 y)-42504 f(x+7 y)+134596 f(x+6 y) \\
& -346104 f(x+5 y)+735471 f(x+4 y)-1307504 f(x+3 y) \\
& +1961256 f(x+2 y)-2496144 f(x+y)+2704156 f(x) \\
& -2496144 f(x-y)+1961256 f(x-2 y)-1307504 f(x-3 y) \\
& +735471 f(x-4 y)-346104 f(x-5 y)+134596 f(x-6 y) \\
& -42504 f(x-7 y)+10626 f(x-8 y)-2024 f(x-9 y) \\
& +276 f(x-10 y)-24 f(x-11 y)+f(x-12 y)=24 ! f(y)
\end{aligned}
$$

where $24 !=620448401733239439360000$.

Now, we will recall the fundamental result in fixed point theory. 
Theorem 1.1. [23] (The alternative of fixed point) Suppose that for a complete generalized metric space $(X, d)$ and a strictly contractive mapping $T: X \rightarrow X$ with Lipschitz constant L. Then, for each given element $x \in X$, either

$$
\left(F_{1}\right) \quad d\left(T^{n} x, T^{n+1} x\right)=\infty \quad \forall n \geq 0,
$$

or

$\left(F_{2}\right)$ there exists a natural number $n_{0}$ such that:

(FPC1) $d\left(T^{n} x, T^{n+1} x\right)<\infty$ for all $n \geq n_{0}$;

(FPC2)The sequence $\left(T^{n} x\right)$ is convergent to a fixed point $y^{*}$ of $T$

(FPC3) $y^{*}$ is the unique fixed point of $T$ in the set $Y=\{y \in$ $\left.X: d\left(T^{n_{0}} x, y\right)<\infty\right\}$;

(FPC4) $d\left(y^{*}, y\right) \leq \frac{1}{1-L} d(y, T y)$ for all $y \in Y$.

In Section 2, the solution of the quattuorvigintic functional equation (1.1) is given. In Sections 3 and 4 the generalized Ulam - Hyers stability of a quattuorvigintic functional equation (1.1) in Banach spaces and quasi-beta Banach spaces using Ulam - Hyers and Ulam - Radus methods are respectively established.

\section{Solution of (1.1)}

In this section, the general solution of the quattuorvigintic functional equation (1.1) is given. For this, let us consider $\mathscr{V}_{1}$ and $\mathscr{V}_{2}$ be real vector spaces.

Theorem 2.1. If $f: \mathscr{V}_{1} \rightarrow \mathscr{V}_{2}$ be a mapping satisfying (1.1) for all $x, y \in \mathscr{V}_{1}$ then $f$ is quattuorvigintic.

Proof. Replacing $(x, y)$ by $(0,0) ;(x, x) ;(x,-x)$ in $(1.1)$, we arrive the set of equations

$$
\begin{aligned}
& f(0)=0 \\
& f(13 x)-24 f(12 x)+276 f(11 x)-2024 f(10 x) \\
& +10626 f(9 x)-42504 f(8 x)+134596 f(7 x) \\
& -346104 f(6 x)+735471 f(5 x)-1307504 f(4 x) \\
& +1961256 f(3 x)-2496144 f(2 x)+2704156 f(x) \\
& -2496144 f(0)+1961256 f(-x)-1307504 f(-2 x) \\
& +735471 f(-3 x)-346104 f(-4 x)+134596 f(-5 x) \\
& -42504 f(-6 x)+10626 f(-7 x)-2024 f(-8 x) \\
& +276 f(-9 x)-24 f(-10 x)+f(-11 x) \\
& =(620448401733239439360000) f(x) \\
& f(-11 x)-24 f(-10 x)+276 f(-9 x)-2024 f(-8 x) \\
& +10626 f(-7 x)-42504 f(-6 x)+134596 f(-5 x) \\
& -346104 f(-4 x)+735471 f(-3 x)-1307504 f(-2 x) \\
& +1961256 f(-x)-2496144 f(0)+2704156 f(x) \\
& -2496144 f(2 x)+1961256 f(3 x)-1307504 f(4 x) \\
& +735471 f(5 x)-346104 f(6 x)+134596 f(7 x) \\
& -42504 f(8 x)+10626 f(9 x)-2024 f(10 x) \\
& +276 f(11 x)-24 f(12 x)+f(13 x) \\
& =(620448401733239439360000) f(-x)
\end{aligned}
$$

for all $x \in \mathscr{V}_{1}$. Comparing (2.2) and (2.3), we get the evenness of $f$, that is

$$
f(-x)=f(x)
$$

for all $x \in \mathscr{V}_{1}$.

Replacing $(x, y)$ by $(12 x, x) ;(11 x, x) ;(10 x, x) ;(9 x, x) ;(8 x, x)$; $(7 x, x) ;(6 x, x) ;(5 x, x) ;(4 x, x) ;(3 x, x) ;(2 x, x) ;(0, x) ;(0,2 x)$; in (1.1) and using evenness of $f$, we arrive the following equations

$$
\begin{aligned}
& f(24 x)-24 f(23 x)+276 f(22 x)-2024 f(21 x) \\
& +10626 f(20 x)-42504 f(19 x)+134596 f(18 x) \\
& -346104 f(17 x)+735471 f(16 x)-1307504 f(15 x) \\
& +1961256 f(14 x)-2496144 f(13 x)+2704156 f(12 x) \\
& -2496144 f(11 x)+1961256 f(10 x)-1307504 f(9 x) \\
& +735471 f(8 x)-346104 f(7 x)+134596 f(6 x) \\
& -42504 f(5 x)+10626 f(4 x)-2024 f(3 x)+276 f(2 x) \\
& -620448401733239439360024 f(x)=0
\end{aligned}
$$

$$
\begin{aligned}
& f(23 x)-24 f(22 x)+276 f(21 x)-2024 f(20 x) \\
& +10626 f(19 x)-42504 f(18 x)+134596 f(17 x) \\
& -346104 f(16 x)+735471 f(15 x)-1307504 f(14 x) \\
& +1961256 f(13 x)-2496144 f(12 x)+2704156 f(11 x) \\
& -2496144 f(10 x)+1961256 f(9 x)-1307504 f(8 x) \\
& +735471 f(7 x)-346104 f(6 x)+134596 f(5 x) \\
& -42504 f(4 x)+10626 f(3 x)-2024 f(2 x) \\
& -620448401733239439359723 f(x)=0
\end{aligned}
$$

$$
\begin{aligned}
& f(22 x)-24 f(21 x)+276 f(20 x)-2024 f(19 x) \\
& +10626 f(18 x)-42504 f(17 x)+134596 f(16 x) \\
& -346104 f(15 x)+735471 f(14 x)-1307504 f(13 x) \\
& +1961256 f(12 x)-2496144 f(11 x)+2704156 f(10 x) \\
& -2496144 f(9 x)+1961256 f(8 x)-1307504 f(7 x) \\
& +735471 f(6 x)-346104 f(5 x)+134596 f(4 x) \\
& -42504 f(3 x)+10627 f(2 x) \\
& -620448401733239439362048 f(x)=0
\end{aligned}
$$

$$
\begin{aligned}
& f(21 x)-24 f(20 x)+276 f(19 x)-2024 f(18 x) \\
& +10626 f(17 x)-42504 f(16 x)+134596 f(15 x) \\
& -346104 f(14 x)+735471 f(13 x)-1307504 f(12 x) \\
& +1961256 f(11 x)-2496144 f(10 x)+2704156 f(9 x) \\
& -2496144 f(8 x)+1961256 f(7 x)-1307504 f(6 x) \\
& +735471 f(5 x)-346104 f(4 x)+134597 f(3 x) \\
& -42528 f(2 x)-620448401733239439349098 f(x)=0
\end{aligned}
$$




$$
\begin{aligned}
& f(20 x)-24 f(19 x)+276 f(18 x)-2024 f(17 x) \\
& +10626 f(16 x)-42504 f(15 x)+134596 f(14 x) \\
& -346104 f(13 x)+735471 f(12 x)-1307504 f(11 x) \\
& +1961256 f(10 x)-2496144 f(9 x)+2704156 f(8 x) \\
& -2496144 f(7 x)+1961256 f(6 x)-1307504 f(5 x) \\
& +735472 f(4 x)-346128 f(3 x)+134872 f(2 x) \\
& -620448401733239439404528 f(x)=0
\end{aligned}
$$

$$
\begin{aligned}
& f(19 x)-24 f(18 x)+276 f(17 x)-2024 f(16 x) \\
& +10626 f(15 x)-42504 f(14 x)+134596 f(13 x) \\
& -346104 f(12 x)+735471 f(11 x)-1307504 f(10 x) \\
& +1961256 f(9 x)-2496144 f(8 x)+2704156 f(7 x) \\
& -2496144 f(6 x)+1961257 f(5 x)-1307528 f(4 x) \\
& +735747 f(3 x)-348128 f(2 x) \\
& -620448401733239439214778 f(x)=0
\end{aligned}
$$

$$
\begin{aligned}
& f(18 x)-24 f(17 x)+276 f(16 x)-2024 f(15 x) \\
& +10626 f(14 x)-42504 f(13 x)+134596 f(12 x) \\
& -346104 f(11 x)+735471 f(10 x)-1307504 f(9 x) \\
& +1961256 f(8 x)-2496144 f(7 x)+2704157 f(6 x) \\
& -2496168 f(5 x)+1961532 f(4 x) \\
& -1309528 f(3 x)+746097 f(2 x) \\
& -620448401733239439748608 f(x)=0
\end{aligned}
$$

$$
f(17 x)-24 f(16 x)+276 f(15 x)-2024 f(14 x)
$$$$
+10626 f(13 x)-42504 f(12 x)+134596 f(11 x)
$$$$
-346104 f(10 x)+735471 f(9 x)-1307504 f(8 x)
$$$$
+1961257 f(7 x)-2496168 f(6 x)+2704432 f(5 x)
$$$$
-2498168 f(4 x)+1971882 f(3 x)-1350008 f(2 x)
$$$$
-620448401733239438489933 f(x)=0
$$

$$
\begin{aligned}
& f(16 x)-24 f(15 x)+276 f(14 x)-2024 f(13 x) \\
& +10626 f(12 x)-42504 f(11 x)+134596 f(10 x) \\
& -346104 f(9 x)+735472 f(8 x)-1307528 f(7 x) \\
& +1961532 f(6 x)-2498168 f(5 x)+2714782 f(4 x) \\
& -25388648 f(3 x)+2095852 f(2 x) \\
& -620448401733239441013608 f(x)=0
\end{aligned}
$$

$$
\begin{aligned}
& f(15 x)-24 f(14 x)+276 f(13 x)-2024 f(12 x) \\
& +10626 f(11 x)-42504 f(10 x)+134597 f(9 x) \\
& -346128 f(8 x)+735747 f(7 x)-1309528 f(6 x) \\
& +1971882 f(5 x)-2538648 f(4 x)+2838752 f(3 x) \\
& -2842248 f(2 x)-620448401733239436663273 f(x)=0
\end{aligned}
$$

(2.14)

$$
\begin{aligned}
& f(14 x)-24 f(13 x)+276 f(12 x)-2024 f(11 x) \\
& +10627 f(10 x)-42528 f(9 x)+134872 f(8 x) \\
& -348128 f(7 x)+746097 f(6 x)-1350008 f(5 x) \\
& +2095852 f(4 x)-2842248 f(3 x)+3439627 f(2 x) \\
& -620448401733239443163648 f(x)=0
\end{aligned}
$$

$$
\begin{aligned}
& f(12 x)-24 f(11 x)+276 f(10 x)-2024 f(9 x) \\
& +10626 f(8 x)-42504 f(7 x)+134596 f(6 x) \\
& -346104 f(5 x)+735471 f(4 x)-1307504 f(3 x) \\
& +1961256 f(2 x) \\
& -310224200866619722176144 f(x)
\end{aligned}
$$

$$
\begin{aligned}
& f(24 x)-24 f(22 x)+276 f(20 x)-2024 f(18 x) \\
& +10626 f(16 x)-42504 f(14 x)+134596 f(12 x) \\
& -346104 f(10 x)+735471 f(8 x)-1307504 f(6 x) \\
& +1961256 f(4 x) \\
& -310224200866619722176144 f(2 x)=0
\end{aligned}
$$

for all $x \in \mathscr{V}_{1}$. Subtracting (2.17) from (2.5), we get

$$
\begin{aligned}
& 24 f(23 x)-300 f(22 x)+2040 f(21 x)-10350 f(20 x) \\
& +42504 f(19 x)-136620 f(18 x)+346104 f(17 x) \\
& -724845 f(16 x)+1307504 f(15 x)-2003760 f(14 x) \\
& +2496144 f(13 x)-2569560 f(12 x)+2496144 f(11 x) \\
& -2307630 f(10 x)+1307504 f(9 x)+346104 f(7 x) \\
& -1442100 f(6 x)+42504 f(5 x)+1950630 f(4 x) \\
& +2024 f(3 x)-310224200866619722176420 f(2 x) \\
& -620448401733239439360024 f(x)=0
\end{aligned}
$$

for all $x \in \mathscr{V}_{1}$. Multiplying (2.6) by 24 and subtracting from (2.18), we reach

$$
\begin{aligned}
& 276 f(22 x)-4600 f(21 x)+38226 f(20 x)-212520 f(19 x) \\
& +883476 f(18 x)-2884200 f(17 x)+7581651 f(16 x) \\
& -16343800 f(15 x)+29376336 f(14 x)-44574000 f(13 x) \\
& +57337896 f(12 x)-62403600 f(11 x)+57600096 f(10 x) \\
& -45762640 f(9 x)+31380096 f(8 x)-17305200 f(7 x) \\
& +6864396 f(6 x)-3187800 f(5 x)+2970726 f(4 x) \\
& -253000 f(3 x)-310224200866619722127844 f(2 x) \\
& +15511210043330985983993376 f(x)=0
\end{aligned}
$$

for all $x \in \mathscr{V}_{1}$. Multiplying (2.7) by 276 and subtracting from 
(2.19), we obtain

$$
\begin{aligned}
& 2024 f(21 x)-37950 f(20 x)+346104 f(19 x) \\
& -2049300 f(18 x)+8846904 f(17 x) \\
& -29566845 f(16 x)+79180904 f(15 x) \\
& -173613660 f(14 x)+316297104 f(13 x) \\
& -483968760 f(12 x)+626532144 f(11 x) \\
& -688746960 f(10 x)+643173104 f(9 x) \\
& -509926560 f(8 x)+343565904 f(7 x) \\
& -196125600 f(6 x)+92336904 f(5 x) \\
& -34177770 f(4 x)+11478104 f(3 x) \\
& -310224200866619725060896 f(2 x) \\
& -186754968921705071247918624 f(x)=0
\end{aligned}
$$

for all $x \in \mathscr{V}_{1}$. Multiplying (2.8) by 20247 and subtracting from (2.20), we have

$$
\begin{aligned}
& 10626 f(20 x)-212520 f(19 x)+2047276 f(18 x) \\
& -12660120 f(17 x)+56461251 f(16 x) \\
& -193241400 f(15 x)+526900836 f(14 x) \\
& -1172296200 f(13 x)+2162419336 f(12 x) \\
& -3343050000 f(11 x)+4363448496 f(10 x) \\
& -4830038640 f(9 x)+4542268896 f(8 x) \\
& -3626016240 f(7 x)+2450262496 f(6 x) \\
& -1396256400 f(5 x)+666336726 f(4 x) \\
& -260946224 f(3 x) \\
& +310224200866619638984224 f(2 x) \\
& -144254253402997781696490492976 f(x)=0
\end{aligned}
$$

for all $x \in \mathscr{V}_{1}$. Multiplying (2.9) by 10626 and subtracting from (2.21), we arrive

$$
\begin{aligned}
& 42504 f(19 x)-88550 f(18 x)+8846904 f(17 x) \\
& -56450625 f(16 x)+258406104 f(15 x) \\
& -903316260 f(14 x)+2505404904 f(13 x) \\
& -5652695510 f(12 x)+10550487504 f(11 x) \\
& -16476857760 f(10 x)+21693987504 f(9 x) \\
& -24192092760 f(8 x)+22898009904 f(7 x) \\
& -18390043760 f(6 x)+12497281104 f(5 x) \\
& -7148788746 f(4 x)+3417009904 f(3 x) \\
& -310224200866621072134096 f(2 x) \\
& -80354272508447183979603007504 f(x)=0
\end{aligned}
$$

from (2.22), we get

$$
\begin{aligned}
& 134596 f(18 x)-2884200 f(17 x)+29577471 f(16 x) \\
& -193241400 f(15 x)+903273756 f(14 x) \\
& -3215463480 f(13 x)+9058108906 f(12 x) \\
& -20709971880 f(11 x)+390997292256 f(10 x) \\
& -61667237520 f(9 x)+81904011816 f(8 x) \\
& -92039436720 f(7 x)+87706060816 f(6 x) \\
& -70863986424 f(5 x)+48426381366 f(4 x) \\
& -27855180584 f(3 x) \\
& +310224200866606275301584 f(2 x) \\
& -34406966118116793103987931616 f(x)=0
\end{aligned}
$$

for all $x \in \mathscr{V}_{1}$. Multiplying (2.11) by 134596 and subtracting from (2.23), we obtain

$$
\begin{aligned}
& 346104 f(17 x)-7571025 f(16 x)+79180904 f(15 x) \\
& -526943340 f(14 x)+2505404904 f(13 x) \\
& -9057974310 f(12 x)+25874242104 f(11 x) \\
& -59894162460 f(10 x)+114317570864 f(9 x) \\
& -182073200760 f(8 x)+243931561104 f(7 x) \\
& -276262654756 f(6 x)+265110241704 f(5 x) \\
& -215587979706 f(4 x)+148402050104 f(3 x) \\
& -310224200866706696973396 f(2 x) \\
& -117916839197803888736391573984 f(x)=0
\end{aligned}
$$

for all $x \in \mathscr{V}_{1}$. Multiplying (2.12) by 346104 and subtracting from (2.24), we have

$$
\begin{aligned}
& 735471 f(16 x)-16343800 f(15 x)+173571156 f(14 x) \\
& -1172296200 f(13 x)+5652830106 f(12 x) \\
& -20709971880 f(11 x)+59893816356 f(10 x) \\
& -140231884120 f(9 x)+270459163656 f(8 x) \\
& -434867331624 f(7 x)+587671074716 f(6 x) \\
& -670904491224 f(5 x)+649037957766 f(4 x) \\
& -534074197624 f(3 x) \\
& +310224200866239453804564 f(2 x) \\
& -332656512831284991355511345016 f(x)=0
\end{aligned}
$$

for all $x \in \mathscr{V}_{1}$. Multiplying (2.10) by 42504 and subtracting 
from (2.25), we arrive

$$
\begin{aligned}
& 1307504 f(15 x)-29418840 f(14 x)+316297104 f(13 x) \\
& -2162284740 f(12 x)+10550487504 f(11 x) \\
& -39097638360 f(10 x)+114317570864 f(9 x) \\
& -270459163656 f(8 x)+526781594064 f(7 x) \\
& -854978826856 f(6 x)+1166425625904 f(5 x) \\
& -1347605474556 f(4 x)+1333027785584 f(3 x) \\
& -310224200867780892170856 f(2 x) \\
& +788978319302432336277230634384 f(x)=0
\end{aligned}
$$

for all $x \in \mathscr{V}_{1}$. Multiplying (2.14) by 1307504 and subtracting from (2.26), we get

$$
\begin{aligned}
& 1961256 f(14 x)-44574000 f(13 x) \\
& +484103356 f(12 x)-3343050000 f(11 x) \\
& +16476511656 f(10 x)-61668545024 f(9 x) \\
& +182104580856 f(8 x)-435210551424 f(7 x) \\
& +857234271256 f(6 x)-1411817976624 f(5 x) \\
& +1971686940036 f(4 x)-2378651809424 f(3 x) \\
& +310224200864064641541864 f(2 x) \\
& +1600217086362249832672206734976 f(x)=0
\end{aligned}
$$

for all $x \in \mathscr{V}_{1}$. Multiplying (2.15) by 1961256, using evenness of $f$ and subtracting from (2.27), we obtain

$$
\begin{aligned}
& 2496144 f(13 x)-57203300 f(12 x) \\
& +626532144 f(11 x)-4365755856 f(10 x) \\
& +21739750144 f(9 x)-82413938376 f(8 x) \\
& +247557577344 f(7 x)-606052946576 f(6 x) \\
& +1235893313424 f(5 x)-2138815370076 f(4 x) \\
& +3195724134064 f(3 x) \\
& -310224200870810630633376 f(2 x) \\
& +28170752366951976090013570356864 f(x)=0
\end{aligned}
$$

for all $x \in \mathscr{V}_{1}$. Multiplying (2.2) by 2496144 and subtracting from (2.28), we have

$$
\begin{aligned}
& 2704156 f(12 x)-64899744 f(11 x) \\
& +746347056 f(10 x)-5473211744 f(9 x) \\
& +28734361656 f(8 x)-114937446624 f(7 x) \\
& +363968580976 f(6 x)-935919208224 f(5 x) \\
& +1988828317476 f(4 x)-3535694786624 f(3 x) \\
& -310224200861316177500064 f(2 x) \\
& +4365803792247991305489858025536 f(x)
\end{aligned}
$$

for all $x \in \mathscr{V}_{1}$. Multiplying (2.16) by 2704156 and subtracting from (2.29), we arrive

$$
\begin{aligned}
& -310224200866619719680000 f(2 x) \\
& +5204698426366666226930810880000 f(x)
\end{aligned}
$$

for all $x \in \mathscr{N} 1$. We achieve from (2.29) that

$$
\begin{aligned}
f(2 x) & =\left(\frac{5204698426366666226930810880000}{310224200866619719680000}\right) f(x) \\
& =16777216 f(x) \\
& =2^{24} f(x)
\end{aligned}
$$

for all $x \in \mathscr{V}_{1}$.

\section{Stability Analysis: Banach Space}

In this section, we prove the the generalized Ulam - Hyers stability of a quattuorvigintic functional equation (1.1) in Banach spaces via two different methods. To provide the stability results, let us assume a function $f: \Gamma_{1} \rightarrow \Gamma_{2}$ by

$$
\begin{aligned}
D f_{24}(x, y)= & f(x+12 y)-24 f(x+11 y)+276 f(x+10 y) \\
& -2024 f(x+9 y)+10626 f(x+8 y) \\
& -42504 f(x+7 y)+134596 f(x+6 y) \\
& -346104 f(x+5 y)+735471 f(x+4 y) \\
& -1307504 f(x+3 y)+1961256 f(x+2 y) \\
& -2496144 f(x+y)+2704156 f(x) \\
& -2496144 f(x-y)+1961256 f(x-2 y) \\
& -1307504 f(x-3 y)+735471 f(x-4 y) \\
& -346104 f(x-5 y)+134596 f(x-6 y) \\
& -42504 f(x-7 y)+10626 f(x-8 y) \\
& -2024 f(x-9 y)+276 f(x-10 y) \\
& -24 f(x-11 y)+f(x-12 y)-24 ! f(y)
\end{aligned}
$$

where $24 !=620448401733239439360000$ for all $x, y \in \Gamma_{1}$, where $\Gamma_{1}$ and $\Gamma_{2}$ are normed space and Banach space respectively.

\subsection{Ulam - Hyers Method}

Theorem 3.1. Let $f: \Gamma_{1} \rightarrow \Gamma_{2}$ be a function fulfilling the functional inequality

$$
\left\|D f_{24}(x, y)\right\| \leq \mathscr{V}(x, y)
$$

where $\mathscr{V}: \Gamma_{1}^{2} \rightarrow[0, \infty)$ is a function satisfying the condition

$$
\lim _{s \rightarrow \infty} \frac{\mathscr{V}\left(2^{p s} x, 2^{p s} y\right)}{2^{24 p s}}=0
$$

for all $x, y \in \Gamma_{1}$ with $p= \pm 1$. Then there exists a unique quattuorvigintic function $\mathscr{Q}_{24}: \Gamma_{1} \rightarrow \Gamma_{2}$ by

$$
\mathscr{Q}_{24}(x)=\lim _{s \rightarrow \infty} \frac{f\left(2^{p s} x\right)}{2^{24 p s}}
$$


which satisfies (1.1) and

$$
\left\|f(x)-\mathscr{Q}_{24}(x)\right\| \leq \frac{1}{2^{24}} \sum_{r=\frac{1-p}{2}}^{\infty} \frac{\mathscr{V}_{24}^{D}\left(2^{p r} x, 2^{p r} x\right)}{2^{24 p r}}
$$

for all $x \in \Gamma_{1}$. The function $\mathscr{V}_{24}^{D}\left(2^{p r} x, 2^{p r} x\right)$ defined by

$$
\begin{aligned}
\mathscr{V}_{24}^{D}(x, x)=\mathscr{V} & \left(0,2 \cdot 2^{p r} x\right)+\mathscr{V}\left(12 \cdot 2^{p r} x, 2^{p r} x\right) \\
& +24 \mathscr{V}\left(11 \cdot 2^{p r} x, 2^{p r} x\right) \\
& +276 \mathscr{V}\left(10 \cdot 2^{p r} x, 2^{p r} x\right) \\
& +20247 \mathscr{V}\left(9 \cdot 2^{p r} x, 2^{p r} x\right) \\
& +10626 \mathscr{V}\left(8 \cdot 2^{p r} x, 2^{p r} x\right) \\
& +42504 \mathscr{V}\left(7 \cdot 2^{p r} x, 2^{p r} x\right) \\
& +134596 \mathscr{V}\left(6 \cdot 2^{p r} x, 2^{p r} x\right) \\
& +346104 \mathscr{V}\left(5 \cdot 2^{p r} x, 2^{p r} x\right) \\
& +735471 \mathscr{V}\left(4 \cdot 2^{p r} x, 2^{p r} x\right) \\
& +1307504 \mathscr{V}\left(3 \cdot 2^{p r} x, 2^{p r} x\right) \\
& +1961256 \mathscr{V}\left(2 \cdot 2^{p r} x, 2^{p r} x\right) \\
& +2496144 \mathscr{V}\left(2^{p r} x, 2^{p r} x\right) \\
& +2704156 \mathscr{V}\left(0,2^{p r} x\right)
\end{aligned}
$$

for all $x \in \Gamma_{1}$.

Proof. Replacing $(x, y)$ by $(12 x, x) ;(11 x, x) ;(10 x, x) ;(9 x, x)$; $(8 x, x) ;(7 x, x) ;(6 x, x) ;(5 x, x) ;(4 x, x) ;(3 x, x) ;(2 x, x)$;

$(0, x) ;(0,2 x)$ in $(3.1)$ and using evenness of $f$, and one can achieve from Theorem 2.1, that

$$
\begin{aligned}
=\| & f(2 x)-16777216 f(x) \| \\
=\| & f(2 x)-2^{24} f(x) \| \\
\leq & \mathscr{V}(0,2 x)+\mathscr{V}(12 x, x)+24 \mathscr{V}(11 x, x) \\
& +276 \mathscr{V}(10 x, x)+20247 \mathscr{V}(9 x, x) \\
& +10626 \mathscr{V}(8 x, x)+42504 \mathscr{V}(7 x, x) \\
& +134596 \mathscr{V}(6 x, x)+346104 \mathscr{V}(5 x, x) \\
& +735471 \mathscr{V}(4 x, x)+1307504 \mathscr{V}(3 x, x) \\
& +1961256 \mathscr{V}(2 x, x)+2496144 \mathscr{V}(x, x) \\
& +2704156 \mathscr{V}(0, x)
\end{aligned}
$$

for all $x \in \Gamma_{1}$. Define

$$
\begin{aligned}
\mathscr{V}_{24}^{D}(x, x)=\mathscr{V} & (0,2 x)+\mathscr{V}(12 x, x)+24 \mathscr{V}(11 x, x) \\
& +276 \mathscr{V}(10 x, x)+20247 \mathscr{V}(9 x, x) \\
& +10626 \mathscr{V}(8 x, x)+42504 \mathscr{V}(7 x, x) \\
& +134596 \mathscr{V}(6 x, x)+346104 \mathscr{V}(5 x, x) \\
& +735471 \mathscr{V}(4 x, x)+1307504 \mathscr{V}(3 x, x) \\
& +1961256 \mathscr{V}(2 x, x)+2496144 \mathscr{V}(x, x) \\
& +2704156 \mathscr{V}(0, x)
\end{aligned}
$$

for all $x \in \Gamma_{1}$. Using (3.7) in (3.6), we arrive

$$
\left\|f(2 x)-2^{24} f(x)\right\| \leq \mathscr{V}_{24}^{D}(x, x)
$$

for all $x \in \Gamma_{1}$. It follows from (3.8) that

$$
\left\|\frac{f(2 x)}{2^{24}}-f(x)\right\| \leq \frac{\mathscr{V}_{24}^{D}(x, x)}{2^{24}}
$$

for all $x \in \Gamma_{1}$. Now substitute $x$ by $2 x$ and dividing by $2^{24}$ in (3.9), we get

$$
\left\|\frac{f\left(2^{2} x\right)}{2^{48}}-\frac{f(2 x)}{2^{24}}\right\| \leq \frac{\mathscr{V}_{24}^{D}(2 x, 2 x)}{2^{48}}
$$

for all $x \in \Gamma_{1}$. From (3.9) and (3.10), we have

$$
\left\|\frac{f\left(2^{2} x\right)}{2^{48}}-f(x)\right\|=\frac{1}{2^{24}}\left[\mathscr{V}_{24}^{D}(x, x)+\frac{\mathscr{V}_{24}^{D}(2 x, 2 x)}{2^{24}}\right]
$$

for all $x \in \Gamma_{1}$. Generalizing for a positive integer $s$, we obtain

$$
\left\|\frac{f\left(2^{s} x\right)}{2^{24 s}}-f(x)\right\| \leq \frac{1}{2^{24}} \sum_{r=0}^{s-1} \frac{\mathscr{V}_{24}^{D}\left(2^{r} x, 2^{r} x\right)}{2^{24 r}}
$$

for all $x \in \Gamma_{1}$. To prove the convergence of the sequence

$$
\left\{\frac{f\left(2^{s} x\right)}{2^{24 s}}\right\}
$$

replacing $x$ by $2^{t} x$ and dividing by $2^{24 t}$ in (3.12), for any $s, t>0$, we get that the sequence $\left\{\frac{f\left(2^{s} x\right)}{2^{24 s}}\right\}$ is a Cauchy in $\Gamma_{2}$ and so it converges. Therefore, we see that a mapping $\mathscr{Q}_{24}(x): \Gamma_{1} \rightarrow \Gamma_{2}$ defined by

$$
\mathscr{Q}_{24}(x)=\lim _{s \rightarrow \infty} \frac{f\left(2^{s} x\right)}{2^{24 s}}
$$

for all $x \in \Gamma_{1}$. Letting $s \rightarrow \infty$ in (3.12), we see that (3.4) holds for all $x \in \Gamma_{1}$. In order to show that $\mathscr{Q}_{24}$ satisfies (1.1), replacing $(x, y)$ by $\left(2^{s} x, 2^{s} y\right)$ and dividing by $2^{24 s}$ in (3.1), we have

$$
\begin{aligned}
\left\|\mathscr{Q}_{24}(x, y)\right\| & =\lim _{s \rightarrow \infty} \frac{1}{2^{24 s}}\left\|D f_{24}\left(2^{s} x, 2^{s} y\right)\right\| \\
& \leq \lim _{s \rightarrow \infty} \frac{1}{2^{24 s}} \mathscr{V}\left(2^{s} x, 2^{s} y\right)
\end{aligned}
$$

for all $x, y \in \Gamma_{1}$. Taking the limit as $s$ approaches to infinity in above inequality, we find that the mapping $\mathscr{Q}_{24}$ is a quattuorvigintic mapping satisfying the functional equation (1.1) for all $x, y \in \Gamma_{1}$.

To prove that $\mathscr{Q}_{24}$ is unique, we assume now that there is $\mathscr{Q}_{24}^{\prime}$ as another quattuorvigintic mapping satisfying (1.1) and the inequality (3.4). Then it follows easily that

$$
\mathscr{Q}_{24}\left(2^{s} x\right)=2^{24 s} \mathscr{Q}_{24}, \quad \mathscr{Q}_{24}^{\prime}\left(2^{s} x\right)=2^{24 s} \mathscr{Q}_{24}^{\prime}(x)
$$

for all $x \in \Gamma_{1}$ and all $s \in \mathbb{N}$. Thus

$$
\begin{aligned}
& \left\|\mathscr{Q}_{24}(x)-\mathscr{Q}_{24}^{\prime}(x)\right\| \\
& =\frac{1}{2^{24 s}}\left\|\mathscr{Q}_{24}\left(2^{s} x\right)-\mathscr{Q}_{24}^{\prime}\left(2^{s} x\right)\right\| \\
& \leq \frac{1}{2^{24 s}}\left\{\left\|\mathscr{Q}_{24}\left(2^{s} x\right)-f\left(2^{s} x\right)\right\|+\left\|f\left(2^{s} x\right)-\mathscr{Q}_{24}^{\prime}\left(2^{s} x\right)\right\|\right\} \\
& \leq \frac{1}{2^{24}} \sum_{r=0}^{\infty} \frac{\mathscr{V}\left(2^{r+s} x, 2^{r+s} x\right)}{2^{24(r+s)}}
\end{aligned}
$$


for all $x \in \Gamma_{1}$. Therefore, as $s \rightarrow \infty$ in the above inequality, one establishes

$$
\mathscr{Q}_{24}(x)-\mathscr{Q}_{24}^{\prime}(x)=0
$$

for all $x \in \Gamma_{1}$, completing the proof of the claimed uniqueness of $\mathscr{Q}_{24}$. Hence the theorem holds for $p=1$.

Case (ii): Assume $p=-1$.

Now replacing $x$ by $\frac{x}{2}$ in (3.8), we get

$$
\left\|f(x)-2^{24} f\left(\frac{x}{2}\right)\right\| \leq \mathscr{V}_{24}^{D}\left(\frac{x}{2}, \frac{x}{2}\right)
$$

for all $x \in \Gamma_{1}$. The rest of the proof is similar to that of case $p=1$. Hence for $p=-1$ also the theorem holds. This completes the proof of the theorem.

The following corollary is an immediate consequence of Theorem 3.1 concerning the stabilities of (1.1).

Corollary 3.2. Let $f: \Gamma_{1} \rightarrow \Gamma_{2}$ be a mapping. If there exist real numbers $\phi$ and $\psi$ such that

$$
\left\|D f_{24}(x, y)\right\| \leq \begin{cases}\phi, \\ \phi\{\|x\| \psi+\|y\| \psi\}, & \psi \neq 24 \\ \phi\|x\| \psi\|y\| \psi, & \psi \neq 12\end{cases}
$$

for all $x, y \in \Gamma_{1}$, then there exists a unique quattuorvigintic function $\mathscr{Q}_{24}: \Gamma_{1} \rightarrow \Gamma_{2}$ such that

$$
\left\|f(x)-\mathscr{Q}_{24}(x)\right\| \leq\left\{\begin{array}{c}
\frac{\phi_{C}}{\left|2^{24}-1\right|}, \\
\frac{\phi_{S}|| z|| \psi}{\left|2^{24}-2 \psi\right|}, \\
\frac{\phi_{P}|| z||^{2 \psi}}{\left|2^{24}-2^{2 \psi \mid}\right|},
\end{array}\right.
$$

where

$$
\begin{aligned}
& \phi_{C}=9758910 \phi \\
& \begin{aligned}
& \phi_{S}=\left\{\left(12^{\psi}+24 \cdot 11^{\psi}+276 \cdot 10^{\psi}\right.\right. \\
&+ 20247 \cdot 9^{\psi}+10626 \cdot 8^{\psi} \\
&+42504 \cdot 7^{\psi}+134596 \cdot 6^{\psi} \\
&+346104 \cdot 5^{\psi}+735471 \cdot 4^{\psi} \\
&\left.+1307504 \cdot 3^{\psi}+1961256 \cdot 2^{\psi}\right) \\
&+12255054\}
\end{aligned} \\
& \phi_{P}=\left(12^{\psi}+24 \cdot 11^{\psi}+276 \cdot 10^{\psi}\right. \\
& +20247 \cdot 9^{\psi}+10626 \cdot 8^{\psi} \\
& +42504 \cdot 7^{\psi}+134596 \cdot 6^{\psi} \\
& +346104 \cdot 5^{\psi}+735471 \cdot 4^{\psi} \\
& +
\end{aligned}
$$

for all $x \in \Gamma_{1}$.

\subsection{Ulam - Radus Method}

Theorem 3.3. Let $f: \Gamma_{1} \rightarrow \Gamma_{2}$ be a function fulfilling th functional inequality

$$
\left\|D f_{24}(x, y)\right\| \leq \mathscr{V}(x, y)
$$

where $\mathscr{V}: \Gamma_{1}^{2} \rightarrow[0, \infty)$ is a function satisfying the condition

$$
\lim _{s \rightarrow \infty} \frac{1}{d_{i}^{24 s}} \mathscr{V}\left(d_{i}^{s} x, d_{i}^{s} y\right)=0
$$

where

$$
d_{i}=\left\{\begin{array}{lll}
2 & \text { if } & i=0 \\
\frac{1}{2} & \text { if } & i=1
\end{array}\right.
$$

for all $x, y \in \Gamma_{1}$. Assume that there exists $L=L(i)$ such that the function

$$
\mathscr{V}_{24}^{F}(x, x)=\mathscr{V}_{24}^{D}\left(\frac{x}{2}, \frac{x}{2}\right)
$$

where $\mathscr{V}_{24}^{D}(x, x)$ is defined in (3.5) with the property

$$
\frac{1}{d_{i}^{24}} \mathscr{V}_{24}^{F}\left(d_{i} x, d_{i} x\right)=L \mathscr{V}_{24}^{F}(x, x)
$$

for all $x \in \Gamma_{1}$. Then there exists a unique quattuorvigintic function $\mathscr{Q}_{24}: \Gamma_{1} \rightarrow \Gamma_{2}$ by

$$
\mathscr{Q}_{24}(x)=\lim _{s \rightarrow \infty} \frac{f\left(d_{i}^{s} x\right)}{d_{i}^{24 s}}
$$

which satisfies (1.1) and

$$
\left\|f(x)-\mathscr{Q}_{24}(x)\right\| \leq\left(\frac{L^{1-i}}{1-L}\right) \mathscr{V}_{24}^{F}(x, x)
$$

for all $x \in \Gamma_{1}$.

Proof. Assume a set $\mathscr{S}=\left\{q / q: \Gamma_{1} \rightarrow \Gamma_{2}, q(0)=0\right\}$ and introduce the generalized metric in the set as follows

$$
\inf \left\{\rho \in(0, \infty):\left\|q_{1}(x)-q_{2}(x)\right\| \leq \phi \mathscr{V}_{24}^{F}(x, x), x \in \Gamma_{1}\right\}
$$

It is easy to see that (3.23) is complete with respect to the defined metric. Define $J: \mathscr{S} \rightarrow \mathscr{S}$ by

$$
J q(x)=\frac{1}{d_{i}^{24}} q\left(d_{i} x\right),
$$

for all $x \in \Gamma_{1}$. Now, from (3.23) and $q_{1}, q_{2} \in \mathscr{S}$, we arrive $d\left(J q_{1}, J q_{2}\right) \leq L \phi$ (See Theorem [23]). This implies $J$ is a strictly contractive mapping on $\mathscr{S}$ with Lipschitz constant $L$.

It follows from (3.23), (3.9) and (3.20) for the case $i=0$, we reach

$$
\begin{aligned}
& \left\|\frac{f(2 x)}{2^{24}}-f(x)\right\| \leq \frac{1}{2^{24}} \mathscr{V}_{24}^{D}(x, x) \\
& \Rightarrow\|J f(x)-f(x)\| \leq L^{1-i} \mathscr{V}_{24}^{F}(x, x), x \in \Gamma_{1} .
\end{aligned}
$$


Again, it follows from (3.23), (3.13) and (3.20) for the case $i=1$, we get

$$
\begin{aligned}
& \left\|f(x)-2^{24} f\left(\frac{x}{2}\right)\right\| \leq \mathscr{V}_{24}^{D}\left(\frac{x}{2}, \frac{x}{2}\right) \\
& \Rightarrow\|f(x)-J f(x)\| \leq L^{1-i} \mathscr{W}_{24}^{F}(x, x), x \in \Gamma_{1} .
\end{aligned}
$$

Thus, from (3.24), (3.25) and (3.23), we arrive

$$
\inf \left\{\begin{aligned}
L^{1-i} \in(0, \infty): & \|f(x)-J f(x)\| \\
& \leq L^{1-i} \mathscr{V}_{24}^{F}(x, x),
\end{aligned}\right.
$$

Hence property (FPC1) of Theorem 1.1 holds. It follows from property (FPC2) of Theorem 1.1 that there exists a fixed point $\mathscr{Q}_{24}$ of $J$ in $\mathscr{S}$ such that

$$
\mathscr{Q}_{24}(x)=\lim _{s \rightarrow \infty} \frac{1}{d_{i}^{24 s}} f\left(d_{i}^{s} x\right)
$$

for all $x \in \Gamma_{1}$. In order to show that $\mathscr{Q}_{24}$ satisfies (1.1), replacing $(x, y)$ by $\left(d_{i}^{s} x, d_{i}^{s} y\right)$ and dividing by $d_{i}^{24 s}$ in (3.16), we have

$$
\begin{aligned}
\left\|\mathscr{Q}_{24}(x, y)\right\| & =\lim _{s \rightarrow \infty} \frac{1}{d_{i}^{24 s}}\left\|D f_{24}\left(d_{i}^{s} x, d_{i}^{s} y\right)\right\| \\
& \leq \lim _{s \rightarrow \infty} \frac{1}{d_{i}^{24 s}} \mathscr{V}\left(d_{i}^{s} x, d_{i}^{s} y\right)=0
\end{aligned}
$$

for all $x, y \in \Gamma_{1}$ i.e., $\mathscr{Q}_{24}$ satisfies the functional equation (1.1). By property (FPC3) of Theorem 1.1, $\mathscr{Q}_{24}$ is the unique fixed point of $J$ in the set

$$
\Delta=\left\{\mathscr{Q}_{24} \in \mathscr{S}: d\left(f, \mathscr{Q}_{24}\right)<\infty\right\},
$$

such that

$$
\inf \left\{\phi \in(0, \infty):\left\|f(x)-\mathscr{Q}_{24}(x)\right\| \leq \phi \mathscr{V}_{24}^{F}(x, x), x \in \Gamma_{1}\right\} .
$$

Finally by property (FPC4) of Theorem 1.1, we obtain

$$
\left\|f(x)-\mathscr{Q}_{24}(x)\right\| \leq\|f(x)-J f(x)\| \leq \frac{L^{1-i}}{1-L},
$$

which yields

$$
\inf \left\{\frac{L^{1-i}}{1-L} \in(0, \infty): \begin{array}{r}
\left\|f(x)-\mathscr{Q}_{24}(x)\right\| \\
\leq\left(\frac{L^{1-i}}{1-L}\right) \mathscr{V}_{24}^{F}(x, x)
\end{array}, x \in \Gamma_{1}\right\} .
$$

This completes the proof of the theorem.

The following corollary is an immediate consequence of Theorem 3.3 concerning the stability of (1.1).

Corollary 3.4. Let $f: \Gamma_{1} \rightarrow \Gamma_{2}$ be a mapping. If there exist real numbers $a$ and $\psi$ satisfying (3.14) for all $x, y \in \Gamma_{1}$, then there exists a unique quattuorvigintic function $\mathscr{Q}_{24}: \Gamma_{1} \rightarrow \Gamma_{2}$ such that (3.15) holds for all $x \in \Gamma_{1}$.
Proof. If we define

$$
\mathscr{V}(x, y)=\left\{\begin{array}{l}
\phi, \\
\phi\left\{\|x\|^{\psi}+\|y\|^{\psi}\right\}, \\
\phi\|x\|^{\psi}\|y\|^{\psi},
\end{array}\right.
$$

and replacing $(x, y)$ by $\left(d_{i}^{n} x, d_{i}^{n} y\right)$ in (3.28) and divided by $d_{i}^{n}$, one can see that (3.17) holds for all $x, y \in \Gamma_{1}$. But from (3.19), (3.28) and (3.7), we have

$$
\begin{aligned}
& \mathscr{V}_{24}^{F}(x, x)=\mathscr{V}_{24}^{D}(x, x)\left(\frac{x}{2}, \frac{x}{2}\right) \\
& =\mathscr{V}\left(0,2 \frac{x}{2}\right)+\mathscr{V}\left(12 \frac{x}{2}, \frac{x}{2}\right)+24 \mathscr{V}\left(11 \frac{x}{2}, \frac{x}{2}\right) \\
& +276 \mathscr{V}\left(10 \frac{x}{2}, \frac{x}{2}\right)+20247 \mathscr{V}\left(9 \frac{x}{2}, \frac{x}{2}\right) \\
& +10626 \mathscr{V}\left(8 \frac{x}{2}, \frac{x}{2}\right)+42504 \mathscr{V}\left(7 \frac{x}{2}, \frac{x}{2}\right) \\
& +134596 \mathscr{V}\left(6 \frac{x}{2}, \frac{x}{2}\right)+346104 \mathscr{V}\left(5 \frac{x}{2}, \frac{x}{2}\right) \\
& +735471 \mathscr{V}\left(4 \frac{x}{2}, \frac{x}{2}\right)+1307504 \mathscr{V}\left(3 \frac{x}{2}, \frac{x}{2}\right) \\
& +1961256 \mathscr{V}\left(2 \frac{x}{2}, \frac{x}{2}\right)+2496144 \mathscr{V}\left(\frac{x}{2}, \frac{x}{2}\right) \\
& +2704156 \mathscr{V}\left(0, \frac{x}{2}\right) \\
& 9758910 \phi \text {, } \\
& \frac{\phi\|x\|^{\psi}}{2^{\psi}}\left\{\left(12^{\psi}+24 \cdot 11^{\psi}+276 \cdot 10^{\psi}\right.\right. \\
& +20247 \cdot 9^{\psi}+10626 \cdot 8^{\psi} \\
& +42504 \cdot 7^{\psi}+134596 \cdot 6^{\psi} \\
& +346104 \cdot 5^{\psi}+735471 \cdot 4^{\psi} \\
& \left.+1307504 \cdot 3^{\psi}+1961256 \cdot 2^{\psi}\right) \\
& =\left\{\begin{array}{l}
+12255054\} \\
\phi\|x\|^{2 \psi}
\end{array}\right. \\
& \frac{\phi\|x\|^{2 \psi}}{2^{\psi}}\left(12^{\psi}+24 \cdot 11^{\psi}+276 \cdot 10^{\psi}\right. \\
& +20247 \cdot 9^{\psi}+10626 \cdot 8^{\psi} \\
& +42504 \cdot 7^{\psi}+134596 \cdot 6^{\psi} \\
& +346104 \cdot 5^{\psi}+735471 \cdot 4^{\psi} \\
& +1307504 \cdot 3^{\psi}+1961256 \cdot 2^{\psi} \\
& +2496144 \text { ), } \\
& =\left\{\begin{array}{c}
\phi_{C}, \\
\frac{\phi_{S}}{2 \psi}, \\
\frac{\phi_{P}}{2^{2 \psi}},
\end{array}\right.
\end{aligned}
$$

for all $x \in \Gamma_{1}$. Now, similarly by (3.20), (3.28) and (3.7), we prove

$$
\frac{1}{d_{i}^{24}} \mathscr{V}_{24}^{F}\left(d_{i} x, d_{i} x\right)=\left\{\begin{array}{l}
d_{i}^{-24} \phi_{C} \\
d_{i}^{\psi-24} \phi_{S} \\
d_{i}^{2 \psi-24} \phi_{P}
\end{array}\right.
$$

for all $x \in \Gamma_{1}$. Thus, the inequality (3.22) holds for

(i). $L=d_{i}^{-24}$ if $i=0$ and $L=\frac{1}{d_{i}^{-24}}$ if $i=1$, 
(ii). $L=d_{i}^{\psi-24}$ for $\psi<24$ if $i=0$ and $L=\frac{1}{d_{i}^{\psi-24}}$ for $\psi>24$ if $i=1$,

(iii). $L=d_{i}^{2 \psi-24}$ for $2 \psi>24$ if $i=0$ and $L=\frac{1}{d_{i}^{2 \psi-24}}$ for $2 \psi>24$ if $i=1$.

Now, from (3.22), we prove the following cases for condition $(i)$.

$$
\begin{aligned}
& L=d_{i}^{-24}=2^{-24}, i=0 \\
& \left\|f(x)-\mathscr{Q}_{24}(x)\right\| \\
& \leq\left(\frac{L^{1-i}}{1-L}\right) \mathscr{V}_{24}^{F}(x, x) \\
& =\left(\frac{\left(2^{-24}\right)^{1-0}}{1-2^{-24}}\right) \phi_{C} \\
& =\left(\frac{2^{-24}}{1-2^{-24}}\right) \phi_{C} \\
& =\left(\frac{1}{2^{24}-1}\right) \phi_{C}
\end{aligned}
$$$$
L=\frac{1}{d_{i}^{-24}}=2^{24}, i=1
$$$$
\left\|f(x)-\mathscr{Q}_{24}(x)\right\|
$$$$
\leq\left(\frac{L^{1-i}}{1-L}\right) \mathscr{V}_{24}^{F}(x, x)
$$$$
=\left(\frac{\left(2^{24}\right)^{1-1}}{1-2^{24}}\right) \phi_{C}
$$$$
=\left(\frac{1}{1-2^{24}}\right) \phi_{C}
$$$$
=\left(\frac{1}{1-2^{24}}\right) \phi_{C}
$$

Also, from (3.22), we prove the following cases for condition $(i i)$.

$$
\begin{array}{ll}
L=d_{i}^{\psi-24}=2^{\psi-24}, i=0 & L=\frac{1}{d_{i}^{\psi-24}}=\frac{1}{2^{\psi-24}}, i=1 \\
\left\|f(x)-\mathscr{Q}_{24}(x)\right\| & \left\|f(x)-\mathscr{Q}_{24}(x)\right\| \\
\leq\left(\frac{L^{1-i}}{1-L}\right) \mathscr{V}_{24}^{F}(x, x) & \leq\left(\frac{L^{1-i}}{1-L}\right) \mathscr{V}_{24}^{F}(x, x) \\
=\left(\frac{\left(2^{\psi-24}\right)^{1-0}}{1-2^{\psi-24}}\right) \phi_{S} & =\left(\frac{\left(2^{24-b}\right)^{1-1}}{1-2^{24-b}}\right) \phi_{S} \\
=\left(\frac{2^{\psi-24}}{1-2^{\psi-24}}\right) \phi_{S} & =\left(\frac{1}{1-2^{24-b}}\right) \phi_{S} \\
=\left(\frac{2^{\psi}}{2^{24}-2^{\psi}}\right) \phi_{S} & =\left(\frac{2^{\psi}}{2^{\psi-2^{24}}}\right) \phi_{S}
\end{array}
$$

Finally, from (3.22), we prove the following cases for condition $(i i i)$.

$$
\begin{array}{lc}
L=d_{i}^{2 \psi-24} & L=\frac{1}{d_{i}^{2 \psi-24}} \\
=2^{2 \psi-24}, i=0 & =2^{24-2 b}, i=1 \\
\left\|f(x)-\mathscr{Q}_{24}(x)\right\| & \left\|f(x)-\mathscr{Q}_{24}(x)\right\| \\
\leq\left(\frac{L^{1-i}}{1-L}\right) \mathscr{V}_{24}^{F}(x, x) & \leq\left(\frac{L^{1-i}}{1-L}\right) \mathscr{V}_{24}^{F}(x, x) \\
=\left(\frac{\left(2^{2 \psi-24}\right)^{1-0}}{1-2^{2 \psi-24}}\right) \phi_{P} & =\left(\frac{\left(2^{24-2 b}\right)^{1-1}}{1-2^{24-2 b}}\right) \phi_{P} \\
=\left(\frac{2^{2 \psi-24}}{1-6^{2 b-24}}\right) \phi_{P} & =\left(\frac{1}{1-2^{24-2 b}}\right) \phi_{P} \\
=\left(\frac{2^{2 \psi}}{2^{24}-2^{2 \psi}}\right) \phi_{P} & =\left(\frac{2^{2 \psi}}{2^{2 \psi}-2^{24}}\right) \phi_{P}
\end{array}
$$

Hence the proof is complete.

\section{Stability Results : Quasi Beta Banach Spaces}

In this section, we prove the the generalized Ulam - Hyers stability of a quattuorvigintic functional equation (1.1) in Quasi beta Banach spaces via two different methods. To provide the stability results, let us assume a function $f: \Gamma_{1} \rightarrow$
$\Gamma_{2}$ by

$$
\begin{aligned}
D f_{24}(x, y)= & f(x+12 y)-24 f(x+11 y)+276 f(x+10 y) \\
& -2024 f(x+9 y)+10626 f(x+8 y) \\
& -42504 f(x+7 y)+134596 f(x+6 y) \\
& -346104 f(x+5 y)+735471 f(x+4 y) \\
& -1307504 f(x+3 y)+1961256 f(x+2 y) \\
& -2496144 f(x+y)+2704156 f(x) \\
& -2496144 f(x-y)+1961256 f(x-2 y) \\
& -1307504 f(x-3 y)+735471 f(x-4 y) \\
& -346104 f(x-5 y)+134596 f(x-6 y) \\
& -42504 f(x-7 y)+10626 f(x-8 y) \\
& -2024 f(x-9 y)+276 f(x-10 y) \\
& -24 f(x-11 y)+f(x-12 y)-24 ! f(y)
\end{aligned}
$$

where $24 !=620448401733239439360000$ for all $x, y \in \Gamma_{1}$, where $\Gamma_{1}$ and $\Gamma_{2}$ are quasi normed space and quasi beta Banach space respectively.

\subsection{Basic Definitions and Notations}

In this section, we give some basic facts concerning quasi- $\beta$ Normed spaces.

We fix a real number $\beta$ with $0<\beta \leq 1$ and let $\mathbb{K}$ denote either $\mathbb{R}$ or $\mathbb{C}$.

Definition 4.1. Let $X$ be a linear space over $\mathbb{K}$. A quasi$\beta$-norm $\|\cdot\|$ is a real-valued function on $X$ satisfying the following:

(QB1) $\|x\| \geq 0$ for all $x \in X$ and $\|x\|=0$ if and only if $x=0$.

(QB2) $\|\rho x\|=|\rho|^{\beta} .\|x\|$ for all $\rho \in \mathbb{K}$ and all $x \in X$.

(QB3) There is a constant $K \geq 1$ such that

$\|x+y\| \leq K(\|x\|+\|y\|)$

for all $x, y \in X$.

The pair $(X,\|\cdot\|)$ is called quasi- $\beta$-normed space if $\|\cdot\|$ is a quasi- $\beta$-norm on $X$. The smallest possible $K$ is called the modulus of concavity of $\|\cdot\|$.

Definition 4.2. A quasi- $\beta$-Banach space is a complete quasi$\beta$-normed space.

\subsection{Ulam - Hyers Method}

Theorem 4.3. Let $f: \Gamma_{1} \rightarrow \Gamma_{2}$ be a function fulfilling th functional inequality

$$
\left\|D f_{24}(x, y)\right\| \leq \mathscr{V}(x, y)
$$

where $\mathscr{V}: \Gamma_{1}^{2} \rightarrow[0, \infty)$ is a function satisfying the condition

$$
\lim _{s \rightarrow \infty} \frac{\mathscr{V}\left(2^{p s} x, 2^{p s} y\right)}{2^{24 p s}}=0
$$

for all $x, y \in \Gamma_{1}$ with $p= \pm 1$. Then there exists a unique quattuorvigintic function $\mathscr{Q}_{24}: \Gamma_{1} \rightarrow \Gamma_{2}$ by

$$
\mathscr{Q}_{24}(x)=\lim _{s \rightarrow \infty} \frac{f\left(2^{p s} x\right)}{2^{24 p s}}
$$


which satisfies (1.1) and

$$
\left\|f(x)-\mathscr{Q}_{24}(x)\right\| \leq \frac{K^{s-1}}{2^{24 \beta}} \sum_{r=\frac{1-p}{2}}^{\infty} \frac{\mathscr{V}_{24}^{D}\left(2^{p r} x, 2^{p r} x\right)}{2^{24 p r}}
$$

for all $x \in \Gamma_{1}$. The function $\mathscr{V}_{24}^{D}\left(2^{p r} x, 2^{p r} x\right)$ defined by

$$
\begin{aligned}
\mathscr{V}_{24}^{D}\left(\left(2^{p r} x, 2^{p r} x\right)=\right. & K^{13} \mathscr{V}\left(0,2 \cdot 2^{p r} x\right)+K^{13} \mathscr{V}(12 \cdot 2 \\
& +K^{12} 24 \mathscr{V}\left(11 \cdot 2^{p r} x, 2^{p r} x\right) \\
& +K^{11} 276 \mathscr{V}\left(10 \cdot 2^{p r} x, 2^{p r} x\right) \\
& +K^{10} 20247 \mathscr{V}\left(9 \cdot 2^{p r} x, 2^{p r} x\right) \\
& +K^{9} 10626 \mathscr{V}\left(8 \cdot 2^{p r} x, 2^{p r} x\right) \\
& +K^{8} 42504 \mathscr{V}\left(7 \cdot 2^{p r} x, 2^{p r} x\right) \\
& +K^{7} 134596 \mathscr{V}\left(6 \cdot 2^{p r} x, 2^{p r} x\right) \\
& +K^{6} 346104 \mathscr{V}\left(5 \cdot 2^{p r} x, 2^{p r} x\right) \\
& +K^{5} 735471 \mathscr{V}\left(4 \cdot 2^{p r} x, 2^{p r} x\right) \\
& +K^{4} 1307504 \mathscr{V}\left(3 \cdot 2^{p r} x, 2^{p r} x\right) \\
& +K^{3} 1961256 \mathscr{V}\left(2 \cdot 2^{p r} x, 2^{p r} x\right) \\
& +K^{2} 2496144 \mathscr{V}\left(2^{p r} x, 2^{p r} x\right) \\
& +K^{2} 2704156 \mathscr{V}\left(0,2^{p r} x\right)
\end{aligned}
$$

for all $x \in \Gamma_{1}$.

Proof. Replacing $(x, y)$ by $(x, x) ;(12 x, x) ;(11 x, x) ;(10 x, x) ;(9 x, x)$; $(8 x, x) ;(7 x, x) ;(6 x, x) ;(5 x, x) ;(4 x, x) ;(3 x, x)$;

$(2 x, x) ;(0, x) ;(0,2 x)$; in $(4.1)$ and using evenness of $f$,

$$
\begin{aligned}
\| f(13 x)-24 f(12 x)+277 f(11 x)-2048 f(10 x) \\
\quad+10902 f(9 x)-44528 f(8 x)+145222 f(7 x) \\
\quad-388608 f(6 x)+870067 f(5 x)-1653608 f(4 x) \\
+2696727 f(3 x)-3803648 f(2 x) \\
\quad-620448401733239434694588 f(x) \| \leq \mathscr{V}(x, x)
\end{aligned}
$$

$$
\begin{aligned}
\| f(24 x)-24 f(23 x)+276 f(22 x)-2024 f(21 x) \\
\quad+10626 f(20 x)-42504 f(19 x) \\
\quad+134596 f(18 x)-346104 f(17 x) \\
\quad+735471 f(16 x)-1307504 f(15 x) \\
\quad+1961256 f(14 x)-2496144 f(13 x) \\
\quad+2704156 f(12 x)-2496144 f(11 x) \\
+1961256 f(10 x)-1307504 f(9 x) \\
+735471 f(8 x)-346104 f(7 x) \\
+134596 f(6 x)-42504 f(5 x) \\
+10626 f(4 x)-2024 f(3 x)+276 f(2 x) \\
\quad-620448401733239439360024 f(x) \| \leq \mathscr{V}(12 x, x)
\end{aligned}
$$

$$
\begin{aligned}
& \| f(23 x)-24 f(22 x)+276 f(21 x)-2024 f(20 x) \\
& +10626 f(19 x)-42504 f(18 x) \\
& +134596 f(17 x)-346104 f(16 x) \\
& +735471 f(15 x)-1307504 f(14 x) \\
& +1961256 f(13 x)-2496144 f(12 x) \\
& +2704156 f(11 x)-2496144 f(10 x) \\
& +1961256 f(9 x)-1307504 f(8 x) \\
& +735471 f(7 x)-346104 f(6 x) \\
& +134596 f(5 x)-42504 f(4 x) \\
& +10626 f(3 x)-2024 f(2 x) \\
& -620448401733239439359723 f(x) \| \leq \mathscr{V}(11 x, x) \\
& \| f(22 x)-24 f(21 x)+276 f(20 x)-2024 f(19 x) \\
& +10626 f(18 x)-42504 f(17 x) \\
& +134596 f(16 x)-346104 f(15 x) \\
& +735471 f(14 x)-1307504 f(13 x) \\
& +1961256 f(12 x)-2496144 f(11 x) \\
& +2704156 f(10 x)-2496144 f(9 x) \\
& +1961256 f(8 x)-1307504 f(7 x) \\
& +735471 f(6 x)-346104 f(5 x) \\
& +134596 f(4 x)-42504 f(3 x)+10627 f(2 x) \\
& -620448401733239439362048 f(x) \| \leq \mathscr{V}(10 x, x)
\end{aligned}
$$

$$
\begin{aligned}
& \| f(21 x)-24 f(20 x)+276 f(19 x)-2024 f(18 x) \\
& \quad+10626 f(17 x)-42504 f(16 x) \\
& \quad+134596 f(15 x)-346104 f(14 x) \\
& \quad+735471 f(13 x)-1307504 f(12 x) \\
& \quad+1961256 f(11 x)-2496144 f(10 x) \\
& \quad+2704156 f(9 x)-2496144 f(8 x) \\
& + \\
& +1961256 f(7 x)-1307504 f(6 x) \\
& + \\
& +735471 f(5 x)-346104 f(4 x) \\
& +134597 f(3 x)-42528 f(2 x) \\
& \\
& -620448401733239439349098 f(x) \| \leq \mathscr{V}(9 x, x)
\end{aligned}
$$




$$
\begin{aligned}
& \| f(20 x)-24 f(19 x)+276 f(18 x)-2024 f(17 x) \\
& \quad+10626 f(16 x)-42504 f(15 x) \\
& \quad+134596 f(14 x)-346104 f(13 x) \\
& \quad+735471 f(12 x)-1307504 f(11 x) \\
& \quad+1961256 f(10 x)-2496144 f(9 x) \\
& \quad+2704156 f(8 x)-2496144 f(7 x) \\
& \quad+1961256 f(6 x)-1307504 f(5 x) \\
& +735472 f(4 x)-346128 f(3 x)+134872 f(2 x) \\
& \quad-620448401733239439404528 f(x) \| \leq \mathscr{V}(8 x, x)
\end{aligned}
$$

$$
\begin{aligned}
& \| f(19 x)-24 f(18 x)+276 f(17 x)-2024 f(16 x) \\
& \quad+10626 f(15 x)-42504 f(14 x) \\
& \quad+134596 f(13 x)-346104 f(12 x) \\
& \quad+735471 f(11 x)-1307504 f(10 x) \\
& \quad+1961256 f(9 x)-2496144 f(8 x) \\
& \quad+2704156 f(7 x)-2496144 f(6 x) \\
& +1961257 f(5 x)-1307528 f(4 x) \\
& +735747 f(3 x)-348128 f(2 x) \\
& \quad-620448401733239439214778 f(x) \| \leq \mathscr{V}(7 x, x)
\end{aligned}
$$

$$
\begin{aligned}
& \| f(18 x)-24 f(17 x)+276 f(16 x)-2024 f(15 x) \\
& \quad+10626 f(14 x)-42504 f(13 x) \\
& \quad+134596 f(12 x)-346104 f(11 x) \\
& \quad+735471 f(10 x)-1307504 f(9 x) \\
& \quad+1961256 f(8 x)-2496144 f(7 x) \\
& +2704157 f(6 x)-2496168 f(5 x) \\
& +1961532 f(4 x)-1309528 f(3 x)+746097 f(2 x) \\
& -620448401733239439748608 f(x) \| \leq \mathscr{V}(6 x, x)
\end{aligned}
$$

$$
\begin{aligned}
& \| f(17 x)-24 f(16 x)+276 f(15 x)-2024 f(14 x) \\
& \quad+10626 f(13 x)-42504 f(12 x) \\
& \quad+134596 f(11 x)-346104 f(10 x) \\
& \quad+735471 f(9 x)-1307504 f(8 x) \\
& \quad+1961257 f(7 x)-2496168 f(6 x) \\
& \quad+2704432 f(5 x)-2498168 f(4 x) \\
& \quad+1971882 f(3 x)-1350008 f(2 x) \\
& \quad-620448401733239438489933 f(x) \| \leq \mathscr{V}(5 x, x)
\end{aligned}
$$

$$
\begin{aligned}
& \| f(16 x)-24 f(15 x)+276 f(14 x)-2024 f(13 x) \\
& \quad+10626 f(12 x)-42504 f(11 x) \\
& +134596 f(10 x)-346104 f(9 x) \\
& +735472 f(8 x)-1307528 f(7 x) \\
& + \\
& +1961532 f(6 x)-2498168 f(5 x) \\
& +2714782 f(4 x)-25388648 f(3 x)+2095852 f(2 x) \\
& -620448401733239441013608 f(x) \| \leq \mathscr{V}(4 x, x)
\end{aligned}
$$

$$
\begin{aligned}
& \| f(15 x)-24 f(14 x)+276 f(13 x)-2024 f(12 x) \\
& \quad+10626 f(11 x)-42504 f(10 x) \\
& \quad+134597 f(9 x)-346128 f(8 x) \\
& +735747 f(7 x)-1309528 f(6 x) \\
& \quad+1971882 f(5 x)-2538648 f(4 x) \\
& +2838752 f(3 x)-2842248 f(2 x) \\
& -620448401733239436663273 f(x) \| \leq \mathscr{V}(3 x, x)
\end{aligned}
$$

$$
\begin{aligned}
& \| f(14 x)-24 f(13 x)+276 f(12 x)-2024 f(11 x) \\
& \quad+10627 f(10 x)-42528 f(9 x) \\
& +134872 f(8 x)-348128 f(7 x) \\
& +746097 f(6 x)-1350008 f(5 x) \\
& +2095852 f(4 x)-2842248 f(3 x)+3439627 f(2 x) \\
& \quad-620448401733239443163648 f(x) \| \leq \mathscr{V}(2 x, x)
\end{aligned}
$$

$$
\begin{aligned}
& \| f(12 x)-24 f(11 x)+276 f(10 x)-2024 f(9 x) \\
& \quad+10626 f(8 x)-42504 f(7 x) \\
& \quad+134596 f(6 x)-346104 f(5 x) \\
& \quad+735471 f(4 x)-1307504 f(3 x)+1961256 f(2 x) \\
& \quad-310224200866619722176144 f(x) \| \leq \mathscr{V}(0, x)
\end{aligned}
$$

$$
\begin{aligned}
& \| f(24 x)-24 f(22 x)+276 f(20 x)-2024 f(18 x) \\
& \quad+10626 f(16 x)-42504 f(14 x) \\
& \quad+134596 f(12 x)-346104 f(10 x) \\
& \quad+735471 f(8 x)-1307504 f(6 x) \\
& \quad+1961256 f(4 x) \\
& \quad-310224200866619722176144 f(2 x) \| \leq \mathscr{V}(0,2 x)
\end{aligned}
$$


for all $x \in \Gamma_{1}$. Combining (4.19) and (4.7), we get

$$
\begin{aligned}
\| & 24 f(23 x)-300 f(22 x)+2040 f(21 x)-10350 f(20 x) \\
& +42504 f(19 x)-136620 f(18 x)+346104 f(17 x) \\
& -724845 f(16 x)+1307504 f(15 x)-2003760 f(14 x) \\
& +2496144 f(13 x)-2569560 f(12 x)+2496144 f(11 x) \\
& -2307630 f(10 x)+1307504 f(9 x)+346104 f(7 x) \\
& -1442100 f(6 x)+42504 f(5 x)+1950630 f(4 x) \\
& +2024 f(3 x)-310224200866619722176420 f(2 x) \\
& -620448401733239439360024 f(x) \| \\
\leq & K(\mathscr{V}(0,2 x)+\mathscr{V}(12 x, x))
\end{aligned}
$$

for all $x \in \Gamma_{1}$. Multiplying (4.8) by 24 and subtracting from (4.20)

$$
\begin{aligned}
\| 276 f & (22 x)-4600 f(21 x)+38226 f(20 x)-212520 f(19 x) \\
& +883476 f(18 x)-2884200 f(17 x) \\
& +7581651 f(16 x)-16343800 f(15 x) \\
& +29376336 f(14 x)-44574000 f(13 x) \\
& +57337896 f(12 x)-62403600 f(11 x) \\
& +57600096 f(10 x)-45762640 f(9 x) \\
& +31380096 f(8 x)-17305200 f(7 x) \\
& +6864396 f(6 x)-3187800 f(5 x) \\
& +2970726 f(4 x)-253000 f(3 x) \\
& -310224200866619722127844 f(2 x) \\
& +15511210043330985983993376 f(x) \| \\
\leq & K^{2} \mathscr{V}(0,2 x)+K^{2} \mathscr{V}(12 x, x)+K 24 \mathscr{V}(11 x, x)
\end{aligned}
$$

for all $x \in \Gamma_{1}$. Multiplying (4.9) by 276 and subtracting from (4.21)

$$
\begin{array}{rl}
\| 2024 & f(21 x)-37950 f(20 x)+346104 f(19 x) \\
& -2049300 f(18 x)+8846904 f(17 x) \\
& -29566845 f(16 x)+79180904 f(15 x) \\
& -173613660 f(14 x)+316297104 f(13 x) \\
& -483968760 f(12 x)+626532144 f(11 x) \\
& -688746960 f(10 x)+643173104 f(9 x) \\
& -509926560 f(8 x)+343565904 f(7 x) \\
& -196125600 f(6 x)+92336904 f(5 x) \\
& -34177770 f(4 x)+11478104 f(3 x) \\
& -310224200866619725060896 f(2 x) \\
& -186754968921705071247918624 f(x) \| \\
\leq & K^{3} \mathscr{V}(0,2 x)+K^{3} \mathscr{V}(12 x, x) \\
& +K^{2} 24 \mathscr{V}(11 x, x)+K 276 \mathscr{V}(10 x, x)
\end{array}
$$

for all $x \in \Gamma_{1}$. Multiplying (4.10) by 20247 and subtracting from (4.22)

$$
\begin{aligned}
& \mid 10626 f(20 x)-212520 f(19 x)+2047276 f(18 x) \\
&-12660120 f(17 x)+56461251 f(16 x) \\
&-193241400 f(15 x)+526900836 f(14 x) \\
&-1172296200 f(13 x)+2162419336 f(12 x) \\
&- 3343050000 f(11 x)+4363448496 f(10 x) \\
&-4830038640 f(9 x)+4542268896 f(8 x) \\
&-3626016240 f(7 x)+2450262496 f(6 x) \\
&-1396256400 f(5 x)+666336726 f(4 x) \\
&-260946224 f(3 x) \\
&+310224200866619638984224 f(2 x) \\
&-144254253402997781696490492976 f(x) \| \\
& \leq K^{4} \mathscr{V}(0,2 x)+K^{4} \mathscr{V}(12 x, x)+K^{3} 24 \mathscr{V}(11 x, x) \\
&+ K^{2} 276 \mathscr{V}(10 x, x)+K 20247 \mathscr{V}(9 x, x)
\end{aligned}
$$

for all $x \in \Gamma_{1}$. Multiplying (4.11) by 10626 and subtracting from (4.23)

$$
\begin{array}{rl}
\mid 42504 & f(19 x)-88550 f(18 x)+8846904 f(17 x) \\
& -56450625 f(16 x)+258406104 f(15 x) \\
& -903316260 f(14 x)+2505404904 f(13 x) \\
& -5652695510 f(12 x)+10550487504 f(11 x) \\
& -16476857760 f(10 x)+21693987504 f(9 x) \\
& -24192092760 f(8 x)+22898009904 f(7 x) \\
& -18390043760 f(6 x)+12497281104 f(5 x) \\
& -7148788746 f(4 x)+3417009904 f(3 x) \\
& -310224200866621072134096 f(2 x) \\
& -80354272508447183979603007504 f(x) \| \\
\leq & K^{5} \mathscr{V}(0,2 x)+K^{5} \mathscr{V}(12 x, x)+K^{4} 24 \mathscr{V}(11 x, x) \\
& +K^{3} 276 \mathscr{V}(10 x, x)+K^{2} 20247 \mathscr{V}(9 x, x) \\
& +K 10626 \mathscr{V}(8 x, x)
\end{array}
$$

for all $x \in \Gamma_{1}$. Multiplying (4.12) by 42504 and subtracting from (4.24) 


$$
\begin{aligned}
& \| 134596 f(18 x)-2884200 f(17 x)+29577471 f(16 x) \\
&- 193241400 f(15 x)+903273756 f(14 x) \\
&- 3215463480 f(13 x)+9058108906 f(12 x) \\
&- 20709971880 f(11 x)+390997292256 f(10 x) \\
&-61667237520 f(9 x)+81904011816 f(8 x) \\
& \quad-92039436720 f(7 x)+87706060816 f(6 x) \\
&-70863986424 f(5 x)+48426381366 f(4 x) \\
&-27855180584 f(3 x) \\
&+ 310224200866606275301584 f(2 x) \\
&-34406966118116793103987931616 f(x) \| \\
& \leq K^{6} \mathscr{V}(0,2 x)+K^{6} \mathscr{V}(12 x, x)+K^{5} 24 \mathscr{V}(11 x, x) \\
&+ K^{4} 276 \mathscr{V}(10 x, x)+K^{3} 20247 \mathscr{V}(9 x, x) \\
&+ K^{2} 10626 \mathscr{V}(8 x, x)+K 42504 \mathscr{V}(7 x, x)
\end{aligned}
$$

$$
\begin{aligned}
& \| 735471 f(16 x)-16343800 f(15 x)+173571156 f(14 x) \\
&- 1172296200 f(13 x)+5652830106 f(12 x) \\
&- 20709971880 f(11 x)+59893816356 f(10 x) \\
&- 140231884120 f(9 x)+270459163656 f(8 x) \\
&- 434867331624 f(7 x)+587671074716 f(6 x) \\
&-670904491224 f(5 x)+649037957766 f(4 x) \\
&- 534074197624 f(3 x) \\
&+310224200866239453804564 f(2 x) \\
&- 332656512831284991355511345016 f(x) \| \\
& \leq K^{8} \mathscr{V}(0,2 x)+K^{8} \mathscr{V}(12 x, x)+K^{7} 24 \mathscr{V}(11 x, x) \\
&+ K^{6} 276 \mathscr{V}(10 x, x)+K^{5} 20247 \mathscr{V}(9 x, x) \\
&+ K^{4} 10626 \mathscr{V}(8 x, x)+K^{3} 42504 \mathscr{V}(7 x, x) \\
&+ K^{2} 134596 \mathscr{V}(6 x, x)+K 346104 \mathscr{V}(5 x, x) \quad(4.27)
\end{aligned}
$$

for all $x \in \Gamma_{1}$. Multiplying (4.13) by 134596 and subtracting from (4.25)

$$
\begin{aligned}
& \mid 346104 f(17 x)-7571025 f(16 x)+79180904 f(15 x) \\
&- 526943340 f(14 x)+2505404904 f(13 x) \\
&-9057974310 f(12 x)+25874242104 f(11 x) \\
&-59894162460 f(10 x)+114317570864 f(9 x) \\
&-182073200760 f(8 x)+243931561104 f(7 x) \\
&-276262654756 f(6 x)+265110241704 f(5 x) \\
&-215587979706 f(4 x)+148402050104 f(3 x) \\
&-310224200866706696973396 f(2 x) \\
&-117916839197803888736391573984 f(x) \| \\
& \leq K^{7} \mathscr{V}(0,2 x)+K^{7} \mathscr{V}(12 x, x)+K^{6} 24 \mathscr{V}(11 x, x) \\
&+K^{5} 276 \mathscr{V}(10 x, x)+K^{4} 20247 \mathscr{V}(9 x, x) \\
&+K^{3} 10626 \mathscr{V}(8 x, x)+K^{2} 42504 \mathscr{V}(7 x, x) \\
&+K 134596 \mathscr{V}(6 x, x)
\end{aligned}
$$

for all $x \in \Gamma_{1}$. Multiplying (4.15) by 735471 and subtracting from (4.27)

$$
\begin{aligned}
\| 1307504 f(15 x)-29418840 f(14 x)+316297104 f(13 x) \\
\quad-2162284740 f(12 x)+10550487504 f(11 x) \\
\quad-39097638360 f(10 x)+114317570864 f(9 x) \\
-270459163656 f(8 x)+526781594064 f(7 x) \\
-854978826856 f(6 x)+1166425625904 f(5 x) \\
-1347605474556 f(4 x)+1333027785584 f(3 x) \\
-310224200867780892170856 f(2 x) \\
+788978319302432336277230634384 f(x) \| \\
\quad \leq K^{9} \mathscr{V}(0,2 x)+K^{9} \mathscr{V}(12 x, x)+K^{8} 24 \mathscr{V}(11 x, x) \\
\quad+K^{7} 276 \mathscr{V}(10 x, x)+K^{6} 20247 \mathscr{V}(9 x, x) \\
+K^{5} 10626 \mathscr{V}(8 x, x)+K^{4} 42504 \mathscr{V}(7 x, x) \\
+K^{3} 134596 \mathscr{V}(6 x, x)+K^{2} 346104 \mathscr{V}(5 x, x) \\
\quad+K 735471 \mathscr{V}(4 x, x)
\end{aligned}
$$

for all $x \in \Gamma_{1}$. Multiplying (4.14) by 346104 and subtracting from (4.26)

for all $x \in \Gamma_{1}$. Multiplying (4.16) by 1307504 and subtracting 
from (4.28)

$$
\begin{aligned}
\| & 1961256 f(14 x)-44574000 f(13 x)+484103356 f(12 x) \\
& -3343050000 f(11 x)+16476511656 f(10 x) \\
& -61668545024 f(9 x)+182104580856 f(8 x) \\
& -435210551424 f(7 x)+857234271256 f(6 x) \\
& -1411817976624 f(5 x)+1971686940036 f(4 x) \\
& -2378651809424 f(3 x) \\
& +310224200864064641541864 f(2 x) \\
& +1600217086362249832672206734976 f(x) \| \\
& \leq K^{10} \mathscr{V}(0,2 x)+K^{10} \mathscr{V}(12 x, x)+K^{9} 24 \mathscr{V}(11 x, x) \\
& +K^{8} 276 \mathscr{V}(10 x, x)+K^{7} 20247 \mathscr{V}(9 x, x) \\
& +K^{6} 10626 \mathscr{V}(8 x, x)+K^{5} 42504 \mathscr{V}(7 x, x) \\
& +K^{4} 134596 \mathscr{V}(6 x, x)+K^{3} 346104 \mathscr{V}(5 x, x) \\
& +K^{2} 735471 \mathscr{V}(4 x, x)+K 1307504 \mathscr{V}(3 x, x)
\end{aligned}
$$

for all $x \in \Gamma_{1}$. Multiplying (4.17) by 1961256, using evenness of $f$ and subtracting from (4.29)

$$
\begin{aligned}
\| & 2496144 f(13 x)-57203300 f(12 x) \\
& +626532144 f(11 x)-4365755856 f(10 x) \\
& +21739750144 f(9 x)-82413938376 f(8 x) \\
& +247557577344 f(7 x)-606052946576 f(6 x) \\
& +1235893313424 f(5 x)-2138815370076 f(4 x) \\
& +3195724134064 f(3 x) \\
& -310224200870810630633376 f(2 x) \\
& +28170752366951976090013570356864 f(x) \| \\
& \leq K^{11} \mathscr{V}(0,2 x)+K^{11} \mathscr{V}(12 x, x)+K^{10} 24 \mathscr{V}(11 x, x) \\
& +K^{9} 276 \mathscr{V}(10 x, x)+K^{8} 20247 \mathscr{V}(9 x, x) \\
& +K^{7} 10626 \mathscr{V}(8 x, x)+K^{6} 42504 \mathscr{V}(7 x, x) \\
& +K^{5} 134596 \mathscr{V}(6 x, x)+K^{4} 346104 \mathscr{V}(5 x, x) \\
& +K^{3} 735471 \mathscr{V}(4 x, x)+K^{2} 1307504 \mathscr{V}(3 x, x) \\
& +K 1961256 \mathscr{V}(2 x, x)
\end{aligned}
$$

for all $x \in \Gamma_{1}$. Multiplying (4.6) by 2496144 and subtracting from (4.30)

$$
\begin{aligned}
\| & 2704156 f(12 x)-64899744 f(11 x) \\
& +746347056 f(10 x)-5473211744 f(9 x) \\
& +28734361656 f(8 x)-114937446624 f(7 x) \\
& +363968580976 f(6 x)-935919208224 f(5 x) \\
& +1988828317476 f(4 x)-3535694786624 f(3 x) \\
& -310224200861316177500064 f(2 x) \\
& +4365803792247991305489858025536 f(x) \| \\
& \leq K^{12} \mathscr{V}(0,2 x)+K^{12} \mathscr{V}(12 x, x)+K^{11} 24 \mathscr{V}(11 x, x) \\
& +K^{10} 276 \mathscr{V}(10 x, x)+K^{9} 20247 \mathscr{V}(9 x, x) \\
& +K^{8} 10626 \mathscr{V}(8 x, x)+K^{7} 42504 \mathscr{V}(7 x, x) \\
& +K^{6} 134596 \mathscr{V}(6 x, x)+K^{5} 346104 \mathscr{V}(5 x, x) \\
& +K^{4} 735471 \mathscr{V}(4 x, x)+K^{3} 1307504 \mathscr{V}(3 x, x) \\
& +K^{2} 1961256 \mathscr{V}(2 x, x)+K 2496144 \mathscr{V}(x, x)
\end{aligned}
$$

for all $x \in \Gamma_{1}$. Multiplying (4.18) by 2704156 and subtracting from (4.31)

$$
\begin{aligned}
\|- & 310224200866619719680000 f(2 x) \\
+ & 5204698426366666226930810880000 f(x) \| \\
\leq & K^{13} \mathscr{V}(0,2 x)+K^{13} \mathscr{V}(12 x, x)+K^{12} 24 \mathscr{V}(11 x, x) \\
& +K^{11} 276 \mathscr{V}(10 x, x)+K^{10} 20247 \mathscr{V}(9 x, x) \\
& +K^{9} 10626 \mathscr{V}(8 x, x)+K^{8} 42504 \mathscr{V}(7 x, x) \\
+ & K^{7} 134596 \mathscr{V}(6 x, x)+K^{6} 346104 \mathscr{V}(5 x, x) \\
+ & K^{5} 735471 \mathscr{V}(4 x, x)+K^{4} 1307504 \mathscr{V}(3 x, x) \\
+ & K^{3} 1961256 \mathscr{V}(2 x, x)+K^{2} 2496144 \mathscr{V}(x, x) \\
+ & K 2704156 \mathscr{V}(0, x)
\end{aligned}
$$

for all $x \in \Gamma_{1}$. We achieve from (4.31) that and one can achieve from Theorem 2.1, that

$$
\begin{aligned}
&\left\|f(2 x)-\left(\frac{5204698426366666226930810880000}{310224200866619719680000}\right) f(x)\right\| \\
&=\|f(2 x)-16777216 f(x)\| \\
&=\left\|f(2 x)-2^{24} f(x)\right\| \\
& \quad \leq K^{13} \mathscr{V}(0,2 x)+K^{13} \mathscr{V}(12 x, x)+K^{12} 24 \mathscr{V}(11 x, x) \\
& \quad+K^{11} 276 \mathscr{V}(10 x, x)+K^{10} 20247 \mathscr{V}(9 x, x) \\
& \quad+K^{9} 10626 \mathscr{V}(8 x, x)+K^{8} 42504 \mathscr{V}(7 x, x) \\
&+K^{7} 134596 \mathscr{V}(6 x, x)+K^{6} 346104 \mathscr{V}(5 x, x) \\
&+ \\
&+K^{5} 735471 \mathscr{V}(4 x, x)+K^{4} 1307504 \mathscr{V}(3 x, x) \\
&+ K^{3} 1961256 \mathscr{V}(2 x, x)+K^{2} 2496144 \mathscr{V}(x, x) \\
&+K 2704156 \mathscr{V}(0, x) \\
&
\end{aligned}
$$


for all $x \in \Gamma_{1}$. Define

$$
\begin{aligned}
& \mathscr{V}_{24}^{D}(x, x)=K^{13} \mathscr{V}(0,2 x)+K^{13} \mathscr{V}(12 x, x) \\
& +K^{12} 24 \mathscr{V}(11 x, x) \\
& +K^{11} 276 \mathscr{V}(10 x, x)+K^{10} 20247 \mathscr{V}(9 x, x) \\
& +K^{9} 10626 \mathscr{V}(8 x, x)+K^{8} 42504 \mathscr{V}(7 x, x) \\
& +K^{7} 134596 \mathscr{V}(6 x, x)+K^{6} 346104 \mathscr{V}(5 x, x) \\
& +K^{5} 735471 \mathscr{V}(4 x, x)+K^{4} 1307504 \mathscr{V}(3 x, x) \\
& +K^{3} 1961256 \mathscr{V}(2 x, x)+K^{2} 2496144 \mathscr{V}(x, x) \\
& +K 2704156 \mathscr{V}(0, x)
\end{aligned}
$$

for all $x \in \Gamma_{1}$. Using (4.34) in (4.33), we arrive

$$
\left\|f(2 x)-2^{24} f(x)\right\| \leq \mathscr{V}_{24}^{D}(x, x)
$$

for all $x \in \Gamma_{1}$. It follows from (4.35) that

$$
\left\|\frac{f(2 x)}{2^{24}}-f(x)\right\| \leq \frac{\mathscr{V}_{24}^{D}(x, x)}{2^{24 \beta}}
$$

for all $x \in \Gamma_{1}$. Now substitute $x$ by $2 x$ and dividing by $2^{24}$ in (4.36), we get

$$
\left\|\frac{f\left(2^{2} x\right)}{2^{48}}-\frac{f(2 x)}{2^{24}}\right\| \leq \frac{\mathscr{V}_{24}^{D}(2 x, 2 x)}{2^{24+24 \beta}}
$$

for all $x \in \Gamma_{1}$. From (4.36) and (4.37), we have

$$
\left\|\frac{f\left(2^{2} x\right)}{2^{48}}-f(x)\right\|=\frac{K}{2^{24 \beta}}\left[\mathscr{V}_{24}^{D}(x, x)+\frac{\mathscr{V}_{24}^{D}(2 x, 2 x)}{2^{24}}\right]
$$

for all $x \in \Gamma_{1}$. Generalizing, for a positive integer $s$, we obtain

$$
\left\|\frac{f\left(2^{s} x\right)}{2^{24 s}}-f(x)\right\| \leq \frac{K^{s-1}}{2^{24 \beta}} \sum_{r=0}^{s-1} \frac{\mathscr{V}_{24}^{D}\left(2^{r} x, 2^{r} x\right)}{2^{24 r}}
$$

for all $x \in \Gamma_{1}$. The rest of the proof is similar to that of Theorem 3.1. This completes the proof of the theorem.

The following corollary is an immediate consequence of Theorem 4.3 concerning the stabilities of (1.1).

Corollary 4.4. Let $f: \Gamma_{1} \rightarrow \Gamma_{2}$ be a mapping. If there exist real numbers $\phi$ and $\psi$ such that

$$
\left\|D f_{24}(x, y)\right\| \leq \begin{cases}\phi, \\ \phi\left\{\|x\| \psi+\|y\|^{\psi}\right\}, & \psi \neq 24 \\ \phi\|x\| \psi\|y\| \psi, & \psi \neq 12\end{cases}
$$

for all $x, y \in \Gamma_{1}$, then there exists a unique quattuorvigintic function $\mathscr{Q}_{24}: \Gamma_{1} \rightarrow \Gamma_{2}$ such that

$$
\left\|f(x)-\mathscr{Q}_{24}(x)\right\| \leq\left\{\begin{array}{l}
\frac{2^{24} K^{s-1} \phi_{\beta C}}{2^{24 \beta}\left|2^{24}-1\right|}, \\
\frac{2^{24} K^{s-1} \phi_{\beta S}|| z||^{\psi}}{2^{24 \beta}\left|2^{24}-2^{\psi} \beta\right|}, \\
\frac{2^{24} K^{s-1} \phi_{\beta P}|| z||^{2 \psi}}{2^{24 \beta}\left|2^{24}-2^{2 \psi \beta}\right|},
\end{array}\right.
$$

where

$$
\begin{aligned}
\phi_{\beta C} & =\phi\left(2 K^{13}+K^{12} 24+K^{11} 276+K^{10} 20247\right. \\
+ & K^{9} 10626+K^{8} 42504+K^{7} 134596 \\
+ & K^{6} 346104+K^{5} 735471+K^{4} 1307504 \\
+ & \left.K^{3} 1961256+K^{2} 2496144+K 2704156\right), \\
\phi_{\beta S}= & \left(K^{13}\left(12^{\psi \beta}+2^{\psi \beta}+1\right)+K^{12} 24\left(11^{\psi \beta}+1\right)\right. \\
& +K^{11} 276\left(10^{\psi \beta}+1\right)+K^{10} 20247\left(9^{\psi \beta}+1\right) \\
& +K^{9} 10626\left(8^{\psi \beta}+1\right)+K^{8} 42504(7 \psi \beta+1) \\
& +K^{7} 134596\left(6^{\psi \beta}+1\right)+K^{6} 346104(5 \psi \beta+1) \\
& +K^{5} 735471\left(4^{\psi \beta}+1\right)+K^{4} 1307504\left(3^{\psi \beta}+1\right) \\
& \left.+K^{3} 1961256\left(2^{\psi \beta}+1\right)+2 K^{2} 2496144+K 2704156\right), \\
\phi_{\beta P} & =\left(K^{13} 12^{\psi} \psi+K^{12} 2411^{\psi \beta}+K^{11} 27610^{\psi \beta}\right. \\
& +K^{10} 202479 \psi \beta+K^{9} 106268^{\psi \beta}+K^{8} 425047 \psi \beta \\
& +K^{7} 1345966^{\psi \beta}+K^{6} 3461045^{\psi \beta} \\
& +K^{5} 7354714 \psi \beta+K^{4} 13075043 \psi \beta \\
& \left.+K^{3} 19612562^{\psi \beta}+K^{2} 2496144\right),
\end{aligned}
$$

for all $x \in \Gamma_{1}$.

\subsection{Ulam - Radus Method}

The proof of the following theorem and corollary is similar to that of results of Section 3.2.

Theorem 4.5. Let $f: \Gamma_{1} \rightarrow \Gamma_{2}$ be a function fulfilling the functional inequality

$$
\left\|D f_{24}(x, y)\right\| \leq \mathscr{V}(x, y)
$$

where $\mathscr{V}: \Gamma_{1}^{2} \rightarrow[0, \infty)$ is a function satisfying the condition

$$
\lim _{s \rightarrow \infty} \frac{1}{d_{i}^{24 s}} \mathscr{V}\left(d_{i}^{s} x, d_{i}^{s} y\right)=0
$$

with where

$$
d_{i}=\left\{\begin{array}{lll}
2 & \text { if } & i=0 \\
\frac{1}{2} & \text { if } & i=1
\end{array}\right.
$$

for all $x, y \in \Gamma_{1}$. Assume that there exists $L=L(i)$ such that the function

$$
\mathscr{V}_{24}^{F}(x, x)=\mathscr{V}_{24}^{D}\left(\frac{x}{2}, \frac{x}{2}\right)
$$

where $\mathscr{V}_{24}^{D}(x, x)$ is defined in (4.5) with the property

$$
\frac{1}{d_{i}^{24}} \mathscr{V}_{24}^{F}\left(d_{i} x, d_{i} x\right)=L \mathscr{V}_{24}^{F}(x, x)
$$

for all $x \in \Gamma_{1}$. Then there exists a unique quattuorvigintic function $\mathscr{Q}_{24}: \Gamma_{1} \rightarrow \Gamma_{2}$ by

$$
\mathscr{Q}_{24}(x)=\lim _{s \rightarrow \infty} \frac{f\left(d_{i}^{s} x\right)}{d_{i}^{24 s}}
$$


which satisfies (1.1) and

$$
\left\|f(x)-\mathscr{Q}_{24}(x)\right\| \leq\left(\frac{L^{1-i}}{1-L}\right) \mathscr{V}_{24}^{F}(x, x)
$$

for all $x \in \Gamma_{1}$.

Corollary 4.6. Let $f: \Gamma_{1} \rightarrow \Gamma_{2}$ be a mapping. If there exist real numbers $\phi$ and $\psi$ satisfying (4.40) for all $x, y \in \Gamma_{1}$, then there exists a unique quattuorvigintic function $\mathscr{Q}_{24}: \Gamma_{1} \rightarrow \Gamma_{2}$ such that (4.41) holds

$$
\left\|f(x)-\mathscr{Q}_{24}(x)\right\| \leq\left\{\begin{array}{c}
\frac{\phi_{\beta C}}{\left|2^{24}-1\right|}, \\
\frac{\phi_{\beta S}|| z||^{\psi}}{\left|2^{24}-2^{\psi}\right|} \\
\frac{\phi_{\beta P}|| z||^{2 \psi}}{\left|2^{24}-2^{2 \psi \mid}\right|},
\end{array}\right.
$$

where $\phi_{\beta C}, \phi_{\beta S}, \phi_{\beta P}$ are defined in (4.42) for all $x \in \Gamma_{1}$.

\section{References}

[1] J. Aczel and J. Dhombres, Functional Equations in Several Variables, Cambridge Univ, Press, 1989.

[2] T. Aoki, On the stability of the linear transformation in Banach spaces, J. Math. Soc. Japan, 2 (1950), 64-66.

[3] M. Arunkumar, Three Dimensional Quartic Functional Equation In Fuzzy Normed Spaces, Far East Journal of Applied Mathematics, 41(2), (2010), 88-94.

[4] M. Arunkumar, G. Britto Antony Xavier, Functional equation Originating from sum of higher Powers of Arithmetic Progression using Difference Operator is stable in Banach space: Direct and Fixed point Methods, Malaya Journal of Matematik (MJM), Vol 1. Issue 1, (2014), 4960.

[5] M. Arunkumar, Perturbation of $n$ Dimensional AQ mixed type Functional Equation via Banach Spaces and Banach Algebra: Hyers Direct and Alternative Fixed Point Methods, International Journal of Advanced Mathematical Sciences (IJAMS), Vol. 2 (1), (2014), 34-56.

[6] M. Arunkumar, S. Hemalatha, E. Sathya, 2 - Variable $A Q C Q$ - functional equation, International Journal of Advanced Mathematical Sciences, 3 (1) (2015), 65-86 .

[7] M. Arunkumar, A. Bodaghi, J. M. Rassias, E. Sathya, The general Solution and approximations of a Decic type functional equation in various normed spaces, Journal of the Chungcheong Mathematical Society, Vol. 29, No. 2 (2016), 287-328 (http://dx.doi.org/10.14403/jcms.2016.29.2.287).

[8] L. Cadariu, V. Radu, Fixed points and stability for functional equations in probabilistic metric and random normed spaces, Fixed Point Theory and Applications. Article ID 589143,1 8 pages, 2009 (2009).

[9] L. Cadariu, V. Radu, Fixed points and the stability of quadratic functional equations, An. Univ. Timisoara, Ser. Mat. Inform. 41 (2003), 25-48.
[10] L. Cadariu, V. Radu, On the stability of the Cauchy functional equation: A fixed point approach, Grazer Math. Ber. 346 (2004), 43-52.

[11] E. Castillo, A. Iglesias and R. Ruiz-coho, Functional Equations in Applied Sciences, Elsevier, B.V.Amslerdam, 2005.

[12] Y.J. Cho, Madjid Eshaghi Gordji, Somaye Zolfaghari, Solutions and Stability of Generalized Mixed Type QC Functional Equations in Random Normed Spaces, Journal of Inequalities and Applications, doi:10.1155/2010/403101

[13] P. W. Cholewa, Remarks on the stability of functional equations, Aequationes Math., 27 (1984), 76-86.

[14] S. Czerwik, On the stability of the quadratic mappings in normed spaces, Abh. Math. Sem. Univ Hamburg., 62 (1992), 59-64.

[15] S. Czerwik, Functional Equations and Inequalities in Several Variables, World Scientific, River Edge, NJ, 2002.

[16] P. Gavruta, A generalization of the Hyers-Ulam-Rassias stability of approximately additive mappings, J. Math. Anal. Appl., 184 (1994), 431-436.

[17] Hassan Azadi Kenary, Sun Young Jang and Choonkil Park, A fixed point approach to the Hyers-Ulam stability of a functional equation in various normed spaces, Fixed Point Theory and Applications, doi:10.1186/1687-18122011-67.

[18] D.H. Hyers, On the stability of the linear functional equation, Proc.Nat. Acad.Sci.,U.S.A.,27 (1941) 222-224.

[19] D.H. Hyers, G. Isac, Th.M. Rassias, Stability of functional equations in several variables, Birkhauser, Basel, 1998.

[20] S.M. Jung, Hyers-Ulam-Rassias Stability of Functional Equations in Mathematical Analysis, Hadronic Press, Palm Harbor, 2001..

[21] Pl. Kannappan, Functional Equations and Inequalities with Applications, Springer Monographs in Mathematics, 2009.

[22] L. Maligranda, A result of Tosio Aoki about a generalization of Hyers-Ulam stability of additive functions- $a$ question of priority, Aequationes Math., 75 (2008), 289-296.

[23] B.Margolis, J.B.Diaz, A fixed point theorem of the alternative for contractions on a generalized complete metric space, Bull. Amer. Math. Soc. 126 (1968), 305-309.

[24] A.K. Mirmostafaee, M.S. Moslehian, Fuzzy versions of Hyers-Ulam-Rassias theorem, Fuzzy Sets and Systems, Vol. 159, no. 6, (2008), 720-729.

[25] A.K. Mirmostafaee, M. Mirzavaziri, M.S. Moslehian, Fuzzy stability of the Jensen functional equation, Fuzzy Sets and Systems, Vol. 159, no. 6, (2008), 730-738.

[26] M. Mursaleen and S. A. Mohiuddine, On stability of a cubic functional equation in intuitionistic fuzzy normed spaces, Chaos, Solitons Fractals, 42, (2009), 2997-3005.

[27] C. Park, J. R. Lee, An AQCQ-functional equation in 
paranormed spaces, Advances in Difference Equations, doi: 10.1186/1687-1847-2012-63.

[28] J.M. Rassias, On approximately of approximately linear mappings by linear mappings, J. Funct. Anal. USA, 46, (1982) 126-130.

[29] J.M. Rassias, H.M. Kim, Generalized Hyers-Ulam stability for general additive functional equations in quasi$\beta$-normed spaces J. Math. Anal. Appl. 356 (2009), no. 1, 302-309.

[30] J.M. Rassias, K.Ravi, M.Arunkumar and B.V.Senthil Kumar, Ulam Stability of Mixed type Cubic and Additive functional equation, Functional Ulam Notions (F.U.N) Nova Science Publishers, 2010, Chapter 13, 149 - 175.

[31] J.M. Rassias, R. Saadati, G. Sadeghi and J. Vahidi, On nonlinear stability in various random normed spaces, J. of inequalities and applications, 2011, 2011:62.

[32] John M. Rassias, M. Arunkumar, E. Sathya, N. Mahesh Kumar, Solution And Stability of A ACQ Functional Equation In Generalized 2-Normed Spaces, Intern. J. Fuzzy Mathematical Archive, Vol. 7, No. 2, (2015), 213-224.

[33] John M. Rassias, M. Arunkumar, E. Sathya, T. Namachivayam, Various Generalized Ulam - Hyers Stabilities of a Nonic Functional Equation, Tbilisi Mathematical Journal, 9(1) (2016), pp. 159 - 196.

[34] M.J. Rassias, M. Arunkumar, S. Ramamoorthi, Stability of the Leibniz additive-quadratic functional equation in quasi- $\beta$ normed spaces: direct and fixed point methods, Journal of Concrete and Applicable Mathematics, 14 (2014), 22 - 46.

[35] Th.M. Rassias, On the stability of the linear mapping in Banach spaces, Proc.Amer.Math. Soc., 72 (1978), $297-$ 300.

[36] Th.M.Rassias, On a modified Hyers-Ulam sequence, J. Math. Anal. Appl. 158no. 1, (1991), 106-113.

[37] Th.M. Rassias, Functional Equations, Inequalities and Applications, Kluwer Acedamic Publishers, Dordrecht, Bostan London, 2003.

[38] K. Ravi, M. Arunkumar and J.M. Rassias, On the Ulam stability for the orthogonally general EulerLagrange type functional equation, International Journal of Mathematical Sciences, Autumn 2008 Vol.3, No. 08, 36-47.

[39] K. Ravi, J.M. Rassias, M. Arunkumar, R. Kodandan, Stability of a generalized mixed type additive, quadratic, cubic and quartic functional equation, J. Inequal. Pure Appl. Math. 10 (2009), no. 4, Article 114, 29 pp.

[40] F. Skof, Proprieta locali e approssimazione di operatori, Rend. Sem. Mat. Fis. Milano, 53 (1983), 113-129.

[41] S.M. Ulam, Problems in Modern Mathematics, Science Editions, Wiley, New York, 1964.

[42] T.Z. Xu, J.M. Rassias, M.J. Rassias, W.X. Xu, A fixed point approach to the stability of quintic and sextic functional equations in quasi- $\beta$-normed spaces, J. Inequal. Appl. 2010, Art. ID 423231, 23 pp.
[43] T.Z. Xu, J.M. Rassias, Approximate Septic and Octic mappings in quasi- $\beta$-normed spaces, J. Computational Analysis and Applications, Vol.15, No. 6, 1110 - 1119, 2013, copyright 2013 Eudoxus Press, LLC.



$$
\begin{aligned}
& \operatorname{ISSN}(\mathrm{P}): 2319-3786 \\
& \text { Malaya Journal of Matematik } \\
& \text { ISSN(O):2321 - } 5666
\end{aligned}
$$

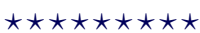

